\title{
STABILITY AND PERSISTENCE IN A MODEL FOR BLUETONGUE DYNAMICS
}

\author{
STEPHEN A. GOURLEY* ${ }^{*}$ HORST R. THIEME ${ }^{\dagger}$, AND P. VAN DEN DRIESSCHE ${ }^{\ddagger}$
}

\begin{abstract}
.
A model for the time evolution of bluetongue, a viral disease in sheep and cattle that is spread by midges as vectors, is formulated as a delay differential equation system of six equations. Midges are assumed to have a pre-adult stage of constant duration, and a general incubation period for bluetongue. A linear stability analysis leads to identification of a basic reproduction number that determines if the disease introduced at a low level dies out, or is uniformly weakly persistent in the midges. Stronger conditions sufficient for global stability of the disease free equilibrium are derived. The control reproduction numbers, which guide control strategies for midges, cattle or sheep, are determined in the special case in which the incubation period for midges is exponentially distributed. The possibility of backward bifurcation is briefly discussed as is an equilibrium situation in which the disease wipes out sheep populations that are introduced in small numbers.
\end{abstract}

Key words. bluetongue, delay, stability, disease persistence, type reproduction number.

AMS subject classifications. 34K12, 34K25, 37C65, 92D30.

1. Introduction. Bluetongue is a viral infection of ruminants, including cattle and sheep, that is spread by biting midges of the Culicoides family. In ruminants it causes changes to the mucous linings of the mouth and nose, but it does not affect humans [7]. According to [7], bluetongue has long been present in most tropical and sub-tropical countries, but since 1999 it has expanded northwards across Europe, with a severe outbreak in the summer of 2007 in Northern Europe. These affected countries started vaccinating livestock in 2008 . The situation on bluetongue disease in the world and latest research is given in the monograph by Mellor et al [22].

A differential equation model for the transmission dynamics of bluetongue has been developed and analysed by Gubbins et al. [11]. This model includes cattle and sheep as separate hosts with infected hosts divided into a number of stages. For the special case of an exponentially distributed infectious period, the basic reproduction number is given, and sensitivity of this number to changes in each parameter is assessed, mainly in the context of UK data. Explicit temperature dependence for some midge parameters is included, as the biting rate, mortality and incubation period of midges is known to be strongly temperature dependent. Hartemink et al. [13] derived an expression for the next generation matrix in a system with three types of individuals. Specifying this to midges, sheep and cattle for bluetongue virus, an expression for the basic reproduction number is derived, and risk maps for an epidemic in the Netherlands are presented.

Here we present a model for bluetongue dynamics that includes midges with a general incubation period as vectors, cattle and sheep as hosts. Our model presumes that midges are active all year round. This is a reasonable assumption for most tropical and subtropical regions, where bluetongue virus is very common (Gibbs and Greiner [10]). It is not a reasonable assumption in regions such as northern Europe where midges would be unlikely to survive the winter. For such regions it would be essential to incorporate the effects of seasonality. An interesting and unresolved

\footnotetext{
*Department of Mathematics, University of Surrey, Guildford, Surrey GU2 7XH, U.K.(s.gourley@surrey.ac.uk).,

†School of Mathematical and Statistical Sciences, Arizona State University, Tempe, AZ 852871804, USA (thieme@math.asu.edu). The work of this author was partly supported by NSF grant DMS-0715451.

${ }^{\ddagger}$ Department of Mathematics and Statistics, University of Victoria, Victoria, B.C., Canada V8W 3R4 (pvdd@math.uvic.ca). The work of this author was partly supported by NSERC and MITACS.
} 
issue in such regions, but not one we consider here, is the precise mechanism for overwintering of the virus. Our model does not incorporate seasonal effects and does not explicitly incorporate temperature dependence. Details of the epidemiology of bluetongue and of our assumptions are given in Section 2, where the model with a general incubation period for midges is formulated. Section 3 contains analysis of the general model, including derivation of a threshold condition in terms of a basic reproduction number that determines whether the disease introduced at a low level dies out, or is uniformly weakly persistent in the midges.

Stronger conditions for global stability of the disease free equilibrium are derived. Further analysis of the special case in which the incubation period is assumed to be exponentially distributed is given in Section 4, which includes derivation of type reproduction numbers and the discussion of a disease equilibrium at which the disease (present in midges and cattle) wipes out sheep populations that are introduced in small numbers. Section 5 contains a discussion of results for our model of bluetongue dynamics.

2. Model formulation. To develop our model for the spread of bluetongue, we consider the time evolution of the disease in the hosts (cattle and sheep) and vectors (midges). The various contact rates, which are determined by the biting rate of the midges and the transmission probabilities, are denoted by $\beta$ with selfexplanatory subscripts, for example, $\beta_{m c}$ is the contact rate from midges to cattle, and includes the probability of transmission of bluetongue from midges to cattle and the biting rate of midges on cattle. The cross infection between hosts (cattle and sheep) and vectors (midges) is modeled using mass action normalized by the total host density. This reservoir frequency-dependent transmission, which assumes that midge biting is not limited by host density, follows that used by [2] for malaria and by several authors for West Nile virus models, e.g., [5, 31].

In cattle, bluetongue generally does not cause death [1], and we assume that it does not cause birth defects. Thus the total number of cattle is taken as a constant $N_{c}$, so that the numbers $I_{c}$ and $S_{c}$ of infectious and susceptible cattle satisfy

$$
I_{c}+S_{c}=N_{c}
$$

Thus, for the cattle there is effectively just one equation for the time evolution of the disease, namely

$$
I_{c}^{\prime}(t)=\frac{\beta_{m c} S_{c}(t) I_{m}(t)}{I_{s}(t)+S_{s}(t)+N_{c}}-\mu_{c} I_{c}(t)
$$

where $\mu_{c}$ is the per-capita cattle natural death rate and $I_{m}(t), I_{s}(t)$ and $S_{s}(t)$ are the numbers of infectious midges, infectious sheep and susceptible sheep respectively at time $t$. Here and throughout the paper, prime denotes differentiation with respect to $t$. Bluetongue does cause death in sheep, but mild cases usually recover rapidly and completely $[1,23]$. The equations for the sheep take the form

$$
\begin{aligned}
& S_{s}^{\prime}(t)=b_{s}\left(S_{s}(t)\right)-\frac{\beta_{m s} S_{s}(t) I_{m}(t)}{I_{s}(t)+S_{s}(t)+N_{c}}+\gamma I_{s}(t)-\mu_{s} S_{s}(t), \\
& I_{s}^{\prime}(t)=\frac{\beta_{m s} S_{s}(t) I_{m}(t)}{I_{s}(t)+S_{s}(t)+N_{c}}-\gamma I_{s}(t)-\mu_{i s} I_{s}(t),
\end{aligned}
$$

with $\mu_{i s}=\mu_{s}+\delta_{s}$ where $\mu_{s}$ is the per-capita sheep natural death rate, $b_{s}(\cdot)$ is the birth rate for sheep, $\delta_{s}$ is their disease induced death rate, $\gamma$ is the per-capita recovery rate and $\beta_{m s}$ is interpreted similarly to $\beta_{m c}$. We assume that only susceptible sheep have viable offspring, since bluetongue virus tends to cause abortion and congenital anomalies in sheep (Merck Veterinary Manual [23]). It is assumed that infected sheep do not compete for reproductive resources. 
The incubation period for the sheep, which is around 4 to 6 days (Merck Veterinary Manual [23]) could also be included, but for reasons of simplicity in the model we have chosen to incorporate only the longer incubation period for the midges (see below). We are assuming that cattle do not recover from the disease while sheep may do. This again is an approximation to the reality that although cattle do not show clinical symptoms they are the main amplifying hosts and tend to be long term virus reservoirs (Hourrigan and Klingsporn [17]). Indeed, in experiments, bluetongue virus was isolated from the blood of infected cattle for up to 49 days after infection, yet it was not isolated from infected sheep after as little as 11 days (Bonneau et al [4]).

The derivation of the evolution equations for the midges is a little more complicated. For now, let $B(t)$ be the rate at time $t$ at which midges become infected as a result of having bitten an infectious cow or an infectious sheep. An expression for $B(t)$ is presented below. A midge acquires the virus with a blood meal, but there is an extrinsic incubation period of the virus in the midges which is very roughly of the order of 2 weeks [1], but fluctuates considerably with temperature and is therefore weather dependent (Wilson and Mellor [30]). For these reasons we model the incubation delay using a probability density function. During the incubation period the virus multiplies in the gut of the midge and then moves to its salivary glands to be injected into a cow or sheep when it bites. Mathematically speaking, when the midge first acquires the virus it moves from the susceptible $S_{m}$ class into the exposed $E_{m}$ class. After the incubation delay the midge moves from the $E_{m}$ class into the infectious $I_{m}$ class.

Let $\mathcal{F}: \mathbb{R}_{+} \rightarrow \mathbb{R}_{+}$be the sojourn function of the incubation period, i.e., $\mathcal{F}(\eta)$ is the probability of still being in the incubation period $\eta$ time units after having taken a blood meal containing the virus [28, ch.12]. We assume that the sojourn function has a probability density $f: \mathbb{R}_{+} \rightarrow \mathbb{R}_{+}$,

$$
\mathcal{F}(\eta)=\int_{\eta}^{\infty} f(r) d r, \quad \mathcal{F}(0)=1 .
$$

Note that $\mathcal{F}$ is a decreasing function. Of all the exposed midges at time $t$, the number that acquired the infection between times $t-\eta$ and $t-\eta+d \eta$ with $d \eta$ infinitesimal is $B(t-\eta) d \eta e^{-\mu_{e m} \eta} \mathcal{F}(\eta)$, as this quantity is the number $B(t-\eta) d \eta$ that acquired the infection between the times mentioned, multiplied by the probability $e^{-\mu_{e m} \eta}$ of still being alive at time $t$, multiplied by the probability of still being in the exposed class at time $t$. Here $\mu_{e m}$ is the per-capita mortality rate for exposed midges, including natural death and death due to disease. The total number $E_{m}(t)$ of exposed midges at time $t$ is the integral of the above quantity over all possible durations of the incubation period and is therefore given by

$$
E_{m}(t)=\int_{0}^{\infty} B(t-\eta) e^{-\mu_{e m} \eta} \mathcal{F}(\eta) d \eta=\int_{-\infty}^{t} B(\eta) e^{-\mu_{e m}(t-\eta)} \mathcal{F}(t-\eta) d \eta .
$$

By (2.4), $E_{m}$ satisfies

$$
E_{m}^{\prime}(t)=B(t)-\int_{-\infty}^{t} B(\eta) e^{-\mu_{e m}(t-\eta)} f(t-\eta) d \eta-\mu_{e m} E_{m}(t) .
$$

We have incorporated the incubation period for the midges via a distributed delay and, as the above derivation makes clear, this formulation allows for a stochastic element in what is otherwise a deterministic model. Our preference for this type of formulation is that the incubation period exhibits strong temperature dependence. We quoted a figure of about 2 weeks, but Gubbins et al [11] cite data suggesting that the period actually varies from 4 days at $30^{\circ} \mathrm{C}$ to 26 days at $15^{\circ} \mathrm{C}$, see also Wilson 
and Mellor [30]. Such wide variation due to temperature, a stochastically varying parameter, justifies our use of a distributed delay formulation for the incubation period in the midges. For an important particular case, assume that the incubation period is given by the negative exponential distribution $f(\xi)=\alpha e^{-\alpha \xi}$ with $\alpha>0$. In this case (2.6) becomes an ordinary differential equation, and further analysis of this case is given in Section 4 .

Next we need to choose an appropriate expression for the rate $B(t)$ at which midges become infected. A midge may acquire the infection from either a cow or a sheep, and therefore there are two contributions to $B(t)$. We write

$$
B(t)=\frac{\beta_{c m} S_{m}(t) I_{c}(t)}{I_{s}(t)+S_{s}(t)+N_{c}}+\frac{\beta_{s m} S_{m}(t) I_{s}(t)}{I_{s}(t)+S_{s}(t)+N_{c}},
$$

where $\beta_{c m}$ and $\beta_{s m}$ are interpreted similarly to $\beta_{m c}$. Insertion of this expression into (2.6) completes the derivation of a differential equation for the exposed midges. An equation for the number of infectious midges $I_{m}(t)$ is easily obtained from it by picking up the relevant flux term from the $E_{m}$ equation, and inserting a mortality term involving the per-capita mortality $\mu_{i m}$ of infectious midges.

For susceptible midges, we assume that only adult midges can contract the virus, and thus $S_{m}(t)$ denotes the number of susceptible adult midges. Midges go through a life cycle involving various pre-adult (such as larval) stages that we assume to be of exactly the same total duration $\tau$ for each midge. For convenience we group all these pre-adult stages together and call it the larval stage, during which there is per-capita larval mortality $\mu_{l}$. The probability of a midge surviving the larval phase to become an adult is $\exp \left(-\mu_{l} \tau\right)$. Since we could not find any information as to whether and how bluetongue affects midge reproduction, we assume that only susceptible midges reproduce and compete for reproductive resources. Thus if the egg laying rate at time $t$ is a function $b_{m}$ of the total number of adult susceptible midges at that time, then the maturation rate of the midges is $\exp \left(-\mu_{l} \tau\right) b_{m}\left(S_{m}(t-\tau)\right)$. Percapita natural mortality of adult susceptible midges is denoted by $\mu_{m}$. Midges pass from the susceptible class to the exposed class at a rate $B(t)$, given by (2.7). These considerations lead to the following equations for susceptible, exposed and infectious midges:

$$
\begin{aligned}
& S_{m}^{\prime}(t)=e^{-\mu_{l} \tau} b_{m}\left(S_{m}(t-\tau)\right)-\mu_{m} S_{m}(t)-\frac{\beta_{c m} S_{m}(t) I_{c}(t)}{I_{s}(t)+S_{s}(t)+N_{c}} \\
& -\frac{\beta_{s m} S_{m}(t) I_{s}(t)}{I_{s}(t)+S_{s}(t)+N_{c}} \\
& E_{m}^{\prime}(t)=-\mu_{e m} E_{m}(t)+\frac{\beta_{c m} S_{m}(t) I_{c}(t)}{I_{s}(t)+S_{s}(t)+N_{c}}+\frac{\beta_{s m} S_{m}(t) I_{s}(t)}{I_{s}(t)+S_{s}(t)+N_{c}} \\
& -\int_{-\infty}^{t}\left(\frac{\beta_{c m} S_{m}(\eta) I_{c}(\eta)}{I_{s}(\eta)+S_{s}(\eta)+N_{c}}+\frac{\beta_{s m} S_{m}(\eta) I_{s}(\eta)}{I_{s}(\eta)+S_{s}(\eta)+N_{c}}\right) \\
& \times e^{-\mu_{e m}(t-\eta)} f(t-\eta) d \eta, \\
& I_{m}^{\prime}(t)=-\mu_{i m} I_{m}(t)+\int_{-\infty}^{t}\left(\frac{\beta_{c m} S_{m}(\eta) I_{c}(\eta)}{I_{s}(\eta)+S_{s}(\eta)+N_{c}}+\frac{\beta_{s m} S_{m}(\eta) I_{s}(\eta)}{I_{s}(\eta)+S_{s}(\eta)+N_{c}}\right) \\
& \times e^{-\mu_{e m}(t-\eta)} f(t-\eta) d \eta \text {. }
\end{aligned}
$$

The model thus consists of six equations, namely $(2.2)$ with $S_{c}(t)=N_{c}-I_{c}(t)$, the two equations of (2.3) and the three equations of (2.8). The system is not fully coupled since the $E_{m}$ equation of (2.8) may be dropped for most purposes and the remaining five equations determine all other variables. Then $E_{m}$ is given by (2.5), with $B(t)$ given by $(2.7)$. 
All parameters are positive with $\mu_{i s} \geq \mu_{s}, \mu_{e m} \geq \mu_{m}$ and $\mu_{i m} \geq \mu_{m}$ except $\tau$, which is nonnegative.

3. Analysis of the model. The model consisting of (2.2), (2.3) and (2.8) is to be solved for $t>0$ subject to initial data, which we now discuss. Because of the delay, initial data for most of the variables have to be prescribed on an interval (in fact for all negative time, for general $f$ ). Moreover, as is common in epidemiological delay models with an exposed class, the components of the initial data are not completely independent of each other. It is possible to prescribe any non-negative initial data for the variables $S_{m}(\theta), S_{s}(\theta), I_{s}(\theta)$ and $I_{c}(\theta), \theta \in(-\infty, 0]$, and any nonnegative value for $I_{m}(0)$. For a well posed problem, $E_{m}(0)$ is determined from (2.5) in terms of the other initial data [6]. The initial data therefore have the form

$$
\begin{aligned}
& I_{m}(0)=I_{m 0} \geq 0, \\
& \begin{array}{l}
S_{m}(\theta)=S_{m 0}(\theta) \geq 0, \\
I_{s}(\theta)=I_{s 0}(\theta) \geq 0,
\end{array} \\
& E_{m}(0)=\int_{-\infty}^{0} S_{m 0}(\eta) \frac{\beta_{c m} I_{c 0}(\eta)+\beta_{s m} I_{s 0}(\eta)}{I_{s 0}(\eta)+S_{s 0}(\eta)+N_{c}} e^{\mu_{e m} \eta} \mathcal{F}(-\eta) d \eta, \\
& S_{s 0}, S_{m 0}, I_{c 0}, I_{s 0} \in C_{\Delta}, \quad 0<3 \Delta<\mu_{e m},
\end{aligned}
$$

with $C_{\Delta}$ defined below, in (3.3). The last assumption in (3.1) needs further explanation. Since the model involves infinite delay, it is important to consider carefully the issue of the choice of an appropriate state space. As noted, the $E_{m}$ equation may be dropped (although at times we still need the $E_{m}$ expression such as in the proof of Proposition 3.4). By the state at time $t$, we mean the entity

$$
S_{t}=\left(S_{s_{t}}, S_{m_{t}}, I_{c_{t}}, I_{s_{t}}, I_{m}(t)\right)
$$

where the subscript $t$ means the state at time $t$, for example $S_{s_{t}}$ is the function defined by $S_{s_{t}}(\theta)=S_{s}(t+\theta), \theta \in(-\infty, 0]$. Note that the $I_{m}$ variable differs from the others in that it does not involve delay. We set up a suitable fading memory space. Let $B U C(-\infty, 0]$ denote the set of bounded uniformly continuous functions on $(-\infty, 0]$. For a $\Delta>0$ to be chosen, let

$$
C_{\Delta}=\left\{\phi:(-\infty, 0] \rightarrow \mathbb{R}: \phi(s) e^{\Delta s} \in B U C(-\infty, 0]\right\}
$$

which is a Banach space with the norm

$$
\|\phi\|=\sup _{s \leq 0}\left|\phi(s) e^{\Delta s}\right| .
$$

Note that, for any $s \leq 0, \phi(s) \leq\|\phi\| e^{-\Delta s}$. The state $S_{t}$ of the solution at time $t$ evolves in the space $C_{\Delta}^{4} \times \mathbb{R}$, where the factor of $\mathbb{R}$ relates to the undelayed variable $I_{m}$. This space is also a Banach space with the norm

$$
\|S\|=\max \left(\left\|S_{s}\right\|,\left\|S_{m}\right\|,\left\|I_{c}\right\|,\left\|I_{s}\right\|,\left|I_{m}\right|\right) .
$$

Regarding the choice of $\Delta$, we examine the delay term in the right hand side of the $I_{m}$ equation of (2.8), and consider the situation at time $t=0$. At this time the integral is the sum of two similar terms the first of which is bounded by

$$
\frac{\beta_{c m}}{N_{c}} \int_{-\infty}^{0} S_{m 0}(\eta) I_{c 0}(\eta) e^{\mu_{e m} \eta} f(-\eta) d \eta
$$


For $S_{m 0}, I_{c 0} \in C_{\Delta}$, this term is bounded by $\left(\beta_{c m}\left\|S_{m 0}\right\|\left\|I_{c 0}\right\| / N_{c}\right) \int_{-\infty}^{0} e^{\left(-2 \Delta+\mu_{e m}\right) \eta}$ $f(-\eta) d \eta$. Finiteness of this integral is assured by choosing $\Delta$ such that $0<2 \Delta<$ $\mu_{e m}$. We assume the stronger condition $0<3 \Delta<\mu_{e m}$ to obtain local existence and uniqueness.

Recall that a function $b: \mathbb{R}_{+} \rightarrow \mathbb{R}$ is locally Lipschitz continuous if for every $S>0$ there exists some $L_{S}>0$ such that $|b(s)-b(\tilde{s})| \leq L_{S}|s-\tilde{s}|$ whenever $0 \leq s, \tilde{s} \leq S$. If $b$ is continuously differentiable on $\mathbb{R}_{+}$, then $b$ is locally Lipschitz continuous. However, our formulation also includes birth functions such as $b(S)=$ $\min \left\{S, S^{\diamond}\right\}$ with some $S^{\diamond}>0$. For the purpose of the next theorem, we extend the birth functions $b_{s}, b_{m}: \mathbb{R}_{+} \rightarrow \mathbb{R}$ from $\mathbb{R}_{+}$to $\mathbb{R}$ by $b_{s}(S)=b_{s}(0)$ and $b_{m}(S)=b_{m}(0)$ for $S<0$. This extension preserves Lipschitz continuity. In realistic cases, $b_{s}(0)=$ $b_{m}(0)=0$.

Theorem 1. If the initial data have the form (3.1) and if $b_{m}\left(S_{m}\right)$ and $b_{s}\left(S_{s}\right)$ are locally Lipschitz continuous, then the model (2.2), (2.3) and (2.8) has a unique solution defined on an interval $[0, A)$ for some $0<A \leq \infty$.

Proof. It is sufficient to prove existence for the subsystem without the $E_{m}$ equation. Let $t^{\sharp}>0$ be fixed but arbitrary. On the interval $t \in\left[0, t^{\sharp}\right]$, the system with

$$
I_{m}^{\prime}(t)=-\mu_{i m} I_{m}(t)+\int_{-\infty}^{t} \frac{\beta_{c m} I_{c}(r)+\beta_{s m} I_{s}(r)}{I_{s}(r)+S_{s}(r)+N_{c}} S_{m}(r) e^{-\mu_{e m}(t-r)} f(t-r) d r
$$

is equivalent to the finite delay system with

$$
\begin{aligned}
I_{m}^{\prime}(t)= & -\mu_{i m} I_{m}(t)+g(t) \\
& +\int_{t-t^{\sharp}}^{t} \frac{\beta_{c m} I_{c}(r)+\beta_{s m} I_{s}(r)}{I_{s}(r)+S_{s}(r)+N_{c}} S_{m}(r) e^{-\mu_{e m}(t-r)} f(t-r) d r,
\end{aligned}
$$

where $g:\left[0, t^{\sharp}\right] \rightarrow \mathbb{R}$ is the given function

$$
g(t)=\int_{-\infty}^{t-t^{\sharp}} \frac{\beta_{c m} I_{c}(r)+\beta_{s m} I_{s}(r)}{I_{s}(r)+S_{s}(r)+N_{c}} S_{m}(r) e^{-\mu_{e m}(t-r)} f(t-r) d r, \quad t \in\left[0, t^{\sharp}\right],
$$

which exists and is continuous if the restrictions of $S_{s}, S_{m}, I_{c}, I_{s}$ to $(-\infty, 0]$ are elements of $C_{\Delta}$. Existence and uniqueness of solutions on subintervals of $\left[0, t^{\sharp}\right]$ follows from standard functional differential equations theory; see for example $[12$, Sec. 3 and 5].

It is of particular importance to know the conditions under which the number of infectious midges $I_{m}(t)$ is strictly positive. The conditions on the initial data that ensure this are enumerated in Proposition 3.1 below. Condition (ii) requires some interpretation. It is in fact the weakest hypothesis on the initial data that ensures that $I_{m}(t)$ becomes positive at some time, when there are no infectious midges at time $t=0$ (i.e., when $I_{m 0}=0$ ). In this situation infectious midges could appear at a positive time if there was a transfer of midges from the susceptible to the exposed compartment prior to time $t=0$, and then some of these midges remained alive and in the exposed class long enough to include the time $t=0$. This could only happen if the function $f(\xi)$, which measures the probability of the incubation period having a particular duration, allows long enough incubation times to permit a midge to enter the infectious compartment at a positive time having become exposed possibly long before time $t=0$. The hypothesis $f\left(\left[-\eta^{*}, \infty\right)\right) \neq\{0\}$ is imposed for this reason. 
Proposition 3.1. Suppose the birth functions $b_{m}\left(S_{m}\right)$ and $b_{s}\left(S_{s}\right)$ are nonnegative and locally Lipschitz continuous. Then each component of any solution of model (2.2), (2.3), (2.8) that satisfies the initial data (3.1) is non-negative wherever the solution exists. If, in addition, either

(i) $I_{m 0}>0$, or

(ii) $S_{m 0}\left(\eta^{*}\right) I_{c 0}\left(\eta^{*}\right)>0$ or $S_{m 0}\left(\eta^{*}\right) I_{s 0}\left(\eta^{*}\right)>0$ for some $\eta^{*} \in(-\infty, 0]$, the initial functions are continuous, $f \in C[0, \infty)$ and $f\left(\left[-\eta^{*}, \infty\right)\right) \neq\{0\}$, or

(iii) $I_{c 0}(0)>0$ or $I_{s 0}(0)>0, S_{m 0}(\theta) \not \equiv 0$ on $[-\tau, 0]$ and $f(\eta)>0$ for all $\eta>0$, then $I_{m}(t)$ becomes strictly positive at some time, and remains strictly positive as long as the solution exists.

Proof. As noted earlier the subsystem without the $E_{m}$ equation consists of a self-consistent subsystem of five equations that determines all variables other than $E_{m}$. We start by establishing non-negativity of all variables other than $E_{m}$ (nonnegativity of $E_{m}$ then follows from (2.5) with (2.7)).

Let $t^{\sharp}>0$ be fixed but arbitrary. To show that the variables other than $E_{m}$, with non-negative initial data, remain non-negative as long as they exist, we use [27, Thm.2.1]. Assume that $S_{s}, S_{m}, I_{c}, I_{s} \geq 0$ on $(-\infty, 0]$ and $I_{m}(0) \geq 0$. Then the function $g(t)$ defined in (3.6) satisfies $g(t) \geq 0$ for $t \in\left[0, t^{\sharp}\right]$. Further, if $S_{s}, S_{m}, I_{c}, I_{s} \geq 0$ and $I_{m}(0)=0$, then the right hand side of (3.5) is non-negative. Similarly, the other assumptions of [27, Thm.2.1] can be verified, and the nonnegativity of every solution defined on a subinterval of $\left[0, t^{\sharp}\right]$ follows.

Strict positivity of $I_{m}(t)$ for all $t>0$ under condition (i) follows as $I_{m}^{\prime}(t) \geq$ $-\mu_{i m} I_{m}(t)$.

Establishing strict positivity of $I_{m}(t)$ under condition (ii) is a little more tricky, as there may not be any infectious midges at time $t=0$. Suppose that $\eta^{*} \in$ $(-\infty, 0]$ is such that $S_{m 0}\left(\eta^{*}\right) I_{c 0}\left(\eta^{*}\right)>0$ or $S_{m 0}\left(\eta^{*}\right) I_{s 0}\left(\eta^{*}\right)>0$. By continuity, $S_{m 0}(\eta) I_{c 0}(\eta)>0$ or $S_{m 0}(\eta) I_{s 0}(\eta)>0$ in some neighborhood of $\eta^{*}$. Now, $f\left(\left[-\eta^{*}, \infty\right)\right) \neq\{0\}$, so there exists $\xi^{*} \geq-\eta^{*}$ such that $f\left(\xi^{*}\right)>0$. Continuity of $f$ implies that $f(\xi)>0$ in some neighborhood of $\xi^{*}$, which can be chosen so that $\xi^{*}>-\eta^{*}$. It also follows that $f\left(\eta^{*}+\xi^{*}-\eta\right)>0$ for $\eta$ sufficiently close to $\eta^{*}$. Now suppose that $I_{m}(t) \equiv 0$ for all $t>0$. Then, the $I_{m}$ equation of (2.8) shows that the first term of its right hand side (the integral term) is zero for all $t>0$. However, this is impossible, because at time $t^{*}=\eta^{*}+\xi^{*}>0$ the integral term becomes

$\int_{-\infty}^{\eta^{*}+\xi^{*}}\left(\frac{\beta_{c m} S_{m}(\eta) I_{c}(\eta)}{I_{s}(\eta)+S_{s}(\eta)+N_{c}}+\frac{\beta_{s m} S_{m}(\eta) I_{s}(\eta)}{I_{s}(\eta)+S_{s}(\eta)+N_{c}}\right) e^{-\mu_{e m}\left(\eta^{*}+\xi^{*}-\eta\right)} f\left(\eta^{*}+\xi^{*}-\eta\right) d \eta$

and the interval of integration includes an interval of $\eta$ for which the integrand is strictly positive. After time $t^{*}, I_{m}(t)$ can never again be zero because $I_{m}^{\prime}(t) \geq$ $-\mu_{i m} I_{m}(t)$, so that $I_{m}(t) \geq I_{m}\left(t^{*}\right) e^{-\mu_{i m}\left(t-t^{*}\right)}>0$.

Now suppose condition (iii) holds, and that $I_{m}(t)=0$ for all $t>0$. Then the third equation of (2.8) shows that $S_{m}(t) I_{c}(t)=S_{m}(t) I_{s}(t)=0$ for all $t>0$. But if $I_{c}$ starts strictly positive then it remains so, and the same is true for $I_{s}$, thus $S_{m}(t)=0$ for all $t>0$. From the $S_{m}$ equation of (2.8) it follows that $S_{m 0}(\theta) \equiv 0$ on $[-\tau, 0]$, a contradiction.

Remark. On inspection of this proof, the need for the compatibility condition in the initial data (3.1) becomes clear, since without this relationship non-negativity of $E_{m}(t)$ need not hold. However any biologically admissible initial data satisfy the compatibility condition.

Lemma 3.2. Let $b: \mathbb{R}_{+} \rightarrow \mathbb{R}_{+}$be continuous. Assume that there are $S^{0}>0$ and $\mu>0$ such that $b(S)<\mu S$ for all $S>S^{0}$. Define the increasing upper hull $\bar{b}$ of $b$ as

$$
\bar{b}(S)=\sup _{0 \leq s \leq S} b(s), \quad b^{0}=\bar{b}\left(S^{0}\right), \quad \breve{S}=b^{0} / \mu .
$$


Then $\bar{b}$ has the following properties:

(a) $\bar{b}$ is monotone increasing and continuous.

(b) $b(S) \leq \bar{b}(S)$ for all $S \geq 0$.

(c) There exist $\breve{S} \geq S^{0}$ such that $\bar{b}(S)<\mu S$ for all $S>\breve{S}$.

Moreover, if $b$ is monotone increasing on $\left[0, S^{0}\right], \breve{S}$ can be chosen as $S^{0}$.

Proof. (a) and (b) are obvious. To show (c), let $S>\breve{S}$. Since $b$ is continuous, $\bar{b}(S)=b(s)$ for some $s \in[0, S]$. We consider the two possibilities:

case $1: s \in\left[0, S^{0}\right]$. Then $\bar{b}(S) \leq \bar{b}\left(S_{0}\right)=b^{0}=\mu \breve{S}<\mu S$.

case $2: s \in\left(S^{0}, S\right]$. Then, by assumption, $\bar{b}(S)=b(s)<\mu s \leq \mu S$.

Now assume that $b$ is monotone increasing on $\left[0, S^{0}\right]$. Then $b^{0}=\bar{b}\left(S^{0}\right)=b\left(S^{0}\right)$ and therefore $\breve{S}=b\left(S^{0}\right) / \mu \leq S^{0}$.

The next result ensures that solutions exist for all positive times and satisfy bounds that are eventually uniform.

Proposition 3.3. Let $b: \mathbb{R}_{+} \rightarrow \mathbb{R}_{+}$be continuous. Assume that there are $S^{0}>0$ and $\mu>0$ such that $b(S)<\mu S$ for all $S>S^{0}$. Choose $\breve{S}$ as in Lemma 3.2. Let $\sigma \in(0, \infty]$ and $N, S:[-\tau, \sigma) \rightarrow \mathbb{R}_{+}$be continuous, $N$ differentiable on $(0, \infty)$, and $S(t) \leq N(t)$ for all $t \in[-\tau, \sigma)$. Let $N$ and $S$ satisfy the differential inequality

$$
N^{\prime}(t) \leq b(S(t-\tau))-\mu N(t), \quad t \in(0, \sigma) .
$$

Then $N(t) \leq \max \left\{\breve{S}, \sup _{\theta \in[-\tau, 0]} N(\theta)\right\}$ for all $t \in[-\tau, \sigma)$. If $\sigma=\infty$, then $\limsup _{t \rightarrow \infty} N(t) \leq \breve{S}$. Moreover, if b is monotone increasing on $\left[0, S^{0}\right]$, then $\breve{S}$ can be replaced by $S^{0}$.

Proof. Let $r \in(0, \sigma)$. Since $N$ is continuous, it is bounded on $[-\tau, r]$ and takes its maximum at some point $t \in[-\tau, r]$. If $t \in[-\tau, 0]$, then $N(t) \leq \sup _{[-\tau, 0]} N$. Assume that $t \in(0, r]$. Then $N^{\prime}(t) \geq 0$ and $N(t) \geq N(t-\tau)$. Suppose $N(t)>\breve{S}$. By the differential inequality,

$$
0 \leq b(S(t-\tau))-\mu N(t) \leq \bar{b}(S(t-\tau))-\mu N(t) .
$$

Since $\bar{b}$ is increasing and $S(t-\tau) \leq N(t-\tau) \leq N(t)$,

$$
0 \leq \bar{b}(N(t-\tau))-\mu N(t) \leq \bar{b}(N(t))-\mu N(t)<0,
$$

with the last inequality following from Lemma 3.2. This contradiction implies that $N(t) \leq \breve{S}$. Since $N$ takes at $t$ its maximum on $[0, r]$, the desired estimate on $[0, r]$ follows. Since $r \in(0, \sigma)$ has been arbitrary, this estimate holds on $[0, \sigma)$.

Now let $\sigma=\infty$. By the fluctuation method [28, Prop.A.22], there exists a sequence $\left(t_{j}\right)$ such that $t_{j} \rightarrow \infty, N\left(t_{j}\right) \rightarrow N^{\infty}=\lim _{\sup _{t \rightarrow \infty}} N(t), N^{\prime}\left(t_{j}\right) \rightarrow 0$ as $j \rightarrow \infty$. Then

$$
0 \leq \limsup _{j \rightarrow \infty} b\left(S\left(t_{j}-\tau\right)\right)-\mu N^{\infty} \leq \limsup _{j \rightarrow \infty} \bar{b}\left(N\left(t_{j}-\tau\right)\right)-\mu N^{\infty} .
$$

Since $\bar{b}$ is increasing,

$$
0 \leq \bar{b}\left(\limsup _{j \rightarrow \infty} N\left(t_{j}-\tau\right)\right)-\mu N^{\infty} \leq \bar{b}\left(N^{\infty}\right)-\mu N^{\infty} .
$$

By Lemma $3.2, N^{\infty} \leq \breve{S}$.

Proposition 3.4. Suppose the birth functions $b_{m}$ and $b_{s}$ are both nonnegative and locally Lipschitz continuous. Assume that there are $S_{m}^{0}>0$ and $S_{s}^{0}>0$ such that

$$
\begin{array}{rc}
e^{-\mu_{l} \tau} b_{m}(S)<\mu_{m} S, & S>S_{m}^{0} \\
b_{s}(S)<\mu_{s} S, & S>S_{s}^{0} .
\end{array}
$$


Then any solution of model (2.2), (2.3), (2.8) that satisfies the initial data (3.1) is defined for all $t \geq 0$ and is bounded. More precisely, there exist constants $\breve{S}_{m}>0$ and $\breve{S}_{s}>0$ that do not depend on the solutions such that

$$
S_{m}(t)+E_{m}(t)+I_{m}(t)=N_{m}(t) \leq \max \left\{\breve{S}_{m}, \sup _{\theta \in[-\tau, 0]} N_{m}(\theta)\right\}
$$

and

$$
S_{s}(t)+I_{s}(t)=N_{s}(t) \leq \max \left\{\breve{S}_{s}, N_{s}(0)\right\}
$$

Moreover

$$
\limsup _{t \rightarrow \infty} N_{m}(t) \leq \breve{S}_{m}, \quad \limsup _{t \rightarrow \infty} N_{s}(t) \leq \breve{S}_{s}
$$

In addition, if $b_{s}$ is monotone increasing on $\left[0, S_{s}^{0}\right]$, then $\breve{S}_{s}$ can be chosen as $S_{s}^{0}$, and if $b_{m}$ is monotone increasing on $\left[0, S_{m}^{0}\right]$, then $S_{m}$ can be chosen as $S_{m}^{0}$.

Proof. The hypotheses include those that by Proposition 3.1 assure the nonnegativity of solution components. Adding the equations of (2.3) and letting $N_{s}=$ $S_{s}+I_{s}$ gives

$$
N_{s}^{\prime}(t)=b_{s}\left(S_{s}(t)\right)-\mu_{s} N_{s}(t)-\delta_{s} I_{s}(t) \leq b_{s}\left(S_{s}(t)\right)-\mu_{s} N_{s}(t) .
$$

By Proposition 3.3, with $\tau=0, N_{s}$, and hence also $S_{s}$ and $I_{s}$, are bounded and the inequalities hold on whatever interval the solution exists.

Adding the equations (2.8) gives

$$
\begin{aligned}
N_{m}^{\prime}(t) & =e^{-\mu_{l} \tau} b_{m}\left(S_{m}(t-\tau)\right)-\mu_{m} S_{m}(t)-\mu_{e m} E_{m}(t)-\mu_{i m} I_{m}(t) \\
& \leq e^{-\mu_{l} \tau} b_{m}\left(S_{m}(t-\tau)\right)-\mu_{m} N_{m}(t)
\end{aligned}
$$

using $\mu_{e m} \geq \mu_{m}$ and $\mu_{i m} \geq \mu_{m}$. By Proposition 3.3, $N_{m}$, and hence $S_{m}, E_{m}$ and $I_{m}$ are bounded and the inequalities hold on whatever interval the solution exists. For the variables $S_{c}$ and $I_{c}$ boundedness is clear.

By [3, Rem.2.1(ii)], the solution exists for all positive times and the estimates hold on $\mathbb{R}_{+}$. The estimates for the superior limits follow from Proposition 3.3.

3.1. The situation with no disease. In the situation when there is no disease, there are $N_{c}$ susceptible cattle, while the numbers $S_{s}$ and $S_{m}$ of susceptible sheep and susceptible midges evolve according to

$$
S_{s}^{\prime}(t)=b_{s}\left(S_{s}(t)\right)-\mu_{s} S_{s}(t)
$$

and

$$
S_{m}^{\prime}(t)=e^{-\mu_{l} \tau} b_{m}\left(S_{m}(t-\tau)\right)-\mu_{m} S_{m}(t) .
$$

A reasonable assumption to make is that when the disease remains absent, numbers of susceptible sheep and midges evolve to constants as $t \rightarrow \infty$. These constants are denoted by $S_{s}^{0}$ and $S_{m}^{0}$, and they must satisfy

$$
b_{s}\left(S_{s}^{0}\right)=\mu_{s} S_{s}^{0}, \quad e^{-\mu_{l} \tau} b_{m}\left(S_{m}^{0}\right)=\mu_{m} S_{m}^{0} .
$$

The existence of unique values $S_{s}^{0}>0$ and $S_{m}^{0}>0$ satisfying these equations depends very much on the functional forms of the birth functions $b_{s}(\cdot)$ and $b_{m}(\cdot)$. We always make the following basic assumptions on these functions, and sometimes additional assumptions as needed. The basic assumptions set out below assure the existence of unique values with $S_{s}^{0}>0$ and $S_{m}^{0}>0$ satisfying (3.9). These values 
correspond to the disease free equilibrium with which we shall be most concerned, but under the assumptions there are four disease free equilibria in all. The others correspond to total extinction, an equilibrium with $S_{s}=S_{s}^{0}>0$ and $S_{m}=0$, and an equilibrium with $S_{m}=S_{m}^{0}>0$ and $S_{s}=0$. For $b_{s}\left(S_{s}\right)$, we assume:

$b_{s}\left(S_{s}\right)$ is non-negative with $b_{s}(0)=0$. Moreover, $b_{s}$ is locally Lipschitz continuous and there exists $S_{s}^{0}>0$ such that $b_{s}\left(S_{s}\right)>\mu_{s} S_{s}$ when $0<S_{s}<S_{s}^{0}$, and $b_{s}\left(S_{s}\right)<\mu_{s} S_{s}$ when $S_{s}>S_{s}^{0}$.

These conditions ensure that (3.7) has zero and $S_{s}^{0}$ as its only equilibria, and that $S_{s}^{0}$ is globally asymptotically stable for all initial data with $S_{s}(0)>0$ as long as no disease is introduced. For $b_{m}\left(S_{m}\right)$, the basic assumptions that we always make are:

$b_{m}\left(S_{m}\right)$ is non-negative with $b_{m}(0)=0$. Moreover, $b_{m}$ is locally Lipschitz continuous and there exists $S_{m}^{0}>0$ such that $e^{-\mu_{l} \tau} b_{m}\left(S_{m}\right)>\mu_{m} S_{m}$ when $0<S_{m}<S_{m}^{0}$, and $e^{-\mu_{l} \tau} b_{m}\left(S_{m}\right)<$ $\mu_{m} S_{m}$ when $S_{m}>S_{m}^{0}$.

Under these assumptions equation (3.8) has zero and $S_{m}^{0}$ as its only equilibria.

The scalar equation (3.8) is very well studied, and various sufficient conditions for the global stability of its equilibria can be found in Kuang [20]. The following result is almost identical to the one in [20, Theorem 9.1, p. 159], but can be proved under less stringent assumptions.

Theorem 2. Suppose that $b_{m}$ satisfies (3.11) and is (not necessarily strictly) monotone increasing on $\left[0, S_{m}^{0}\right]$, and that $S_{m}(\theta) \geq 0$ for $\theta \in[-\tau, 0]$ with $S_{m}(\theta) \not \equiv 0$ on $[-\tau, 0]$. Then the solution of (3.8) satisfies $S_{m}(t) \rightarrow S_{m}^{0}$ as $t \rightarrow \infty$.

Proof. By Proposition 3.3, lim $\sup _{t \rightarrow \infty} S_{m}(t)=S_{m}^{\infty} \leq S_{m}^{0}$. By the fluctuation method [28, Prop.A.22], there exists a sequence $\left(t_{j}\right)$ such that $t_{j} \rightarrow \infty, S_{m}\left(t_{j}\right) \rightarrow$ $S_{m \infty}=\liminf _{t \rightarrow \infty} S_{m}(t), S_{m}^{\prime}\left(t_{j}\right) \rightarrow 0$ as $j \rightarrow \infty$. Then

$0=\lim _{j \rightarrow \infty}\left(e^{-\mu_{l} \tau} b_{m}\left(S_{m}\left(t_{j}-\tau\right)\right)-\mu_{m} S_{m}\left(t_{j}\right)\right) \geq e^{-\mu_{l} \tau} \liminf _{j \rightarrow \infty} b_{m}\left(S_{m}\left(t_{j}-\tau\right)\right)-\mu_{m} S_{m \infty}$.

Now

$$
\liminf _{j \rightarrow \infty} b_{m}\left(S_{m}\left(t_{j}-\tau\right)\right)=b_{m}(S), \quad \text { where } S \in\left[S_{m \infty}, S_{m}^{\infty}\right] \subseteq\left[0, S_{m}^{0}\right] .
$$

Since $b_{m}$ is increasing on $\left[0, S_{m}^{0}\right]$,

$$
\liminf _{j \rightarrow \infty} b_{m}\left(S_{m}\left(t_{j}-\tau\right)\right) \geq b_{m}\left(S_{m \infty}\right) .
$$

Substituting this inequality into a previous one,

$$
0 \geq e^{-\mu_{l} \tau} b_{m}\left(S_{m \infty}\right)-\mu_{m} S_{m \infty}
$$

By (3.11), $S_{m \infty} \geq S_{m}^{0}$ or $S_{m \infty}=0$. It is sufficient to rule out the latter, because if $S_{m \infty} \geq S_{m}^{0}$ then

$$
S_{m}^{\infty} \leq S_{m}^{0} \leq S_{m \infty} \leq S_{m}^{\infty}
$$

which implies $S_{m}^{\infty}=S_{m \infty}=S_{m}^{0}$ and $S_{m}(t) \rightarrow S_{m}^{0}$ as $t \rightarrow \infty$.

We now show that $S_{m \infty} \neq 0$. It follows from the assumptions on the initial data that $S_{m}(t)>0$ for all $t>\tau$. Suppose for contradiction that $S_{m \infty}=0$. For each $n \in \mathbb{N}$, there exists $r_{n} \in[\tau+1, \tau+1+n]$ such that $S_{m}\left(r_{n}\right)=\inf \left\{S_{m}(s) ; \tau+1 \leq\right.$ $s \leq \tau+1+n\}$. Since $S_{m \infty}=0, r_{n} \rightarrow \infty$ as $n \rightarrow \infty$. For sufficiently large $n$, $S_{m}^{\prime}\left(r_{n}\right) \leq 0, S_{m}\left(r_{n}\right)<S_{m}^{0}$, and $S_{m}\left(r_{n}\right) \leq S_{m}\left(r_{n}-\tau\right)$. From (3.8) and (3.11),

$$
S_{m}^{\prime}\left(r_{n}\right) \geq e^{-\mu_{l} \tau} b_{m}\left(S_{m}\left(r_{n}\right)\right)-\mu_{m} S_{m}\left(r_{n}\right)>0,
$$

a contradiction. 
3.2. Local asymptotic stability of the disease-free equilibrium. We have noted the existence of a disease-free equilibrium with $S_{m}=S_{m}^{0}>0, S_{s}=$ $S_{s}^{0}>0, S_{c}=S_{c}^{0}=N_{c}>0$, and the other variables all zero if (3.10) and (3.11) are assumed. There are three other disease-free equilibria, one with $S_{m}=S_{s}=0$, one with $S_{m}=0$ and $S_{s}>0$, and the third with $S_{m}>0$ and $S_{s}=0$. These latter three are all easily seen to be unstable. The cattle-only and the cattle-midge disease-free equilibria are unstable because, if there is no disease and sheep are introduced, the number of sheep converges to $S_{s}^{0}$. The cattle-sheep disease-free equilibrium is unstable under the assumptions of Theorem 2 because, if there is no disease and midges are introduced, the number of midges converges to $S_{m}^{0}$.

To obtain a threshold inequality (the weakest possible) for the local asymptotic stability of the first disease-free equilibrium (with susceptible midges, sheep and cattle positive) in terms of a basic reproduction number, we introduce the following notation,

$$
\begin{gathered}
\mathcal{R}_{c m}=\frac{1}{\mu_{c}} \frac{\beta_{c m} S_{m}^{0}}{\left(S_{s}^{0}+N_{c}\right)} \hat{f}\left(\mu_{e m}\right), \\
\mathcal{R}_{m c}=\frac{1}{\mu_{i m}} \frac{\beta_{m c} N_{c}}{\left(S_{s}^{0}+N_{c}\right)}, \\
\mathcal{R}_{s m}=\frac{1}{\left(\gamma+\mu_{i s}\right)} \frac{\beta_{s m} S_{m}^{0}}{\left(S_{s}^{0}+N_{c}\right)} \hat{f}\left(\mu_{e m}\right), \\
\mathcal{R}_{m s}=\frac{1}{\mu_{i m}} \frac{\beta_{m s} S_{s}^{0}}{\left(S_{s}^{0}+N_{c}\right)} .
\end{gathered}
$$

Let us focus on (3.14) to explain these expressions. Here $\frac{1}{\gamma+\mu_{i s}}$ is the average sojourn of a sheep in the infectious stage. A typical infectious sheep infects susceptible midges at the rate $\frac{\beta_{s m} S_{m}^{0}}{S_{s}^{0}+N_{c}}$. The quantity $\hat{f}\left(\mu_{e m}\right)=\int_{0}^{\infty} e^{-\mu_{e m} t} f(t) d t$ is the probability of an infected midge to survive the latency period and become infectious [28, Sec.13.6]. So $\mathcal{R}_{s m}$ is the average number of infectious midges that is produced by one typical infectious sheep when the bluetongue is introduced at the disease-free equilibrium, i.e., it is the basic reproduction number of the infection from sheep to midges. Similarly, $\mathcal{R}_{m s}$ is the basic reproduction number of the infection from midges to sheep, $\mathcal{R}_{c m}$ is the basic reproduction number of the infection from cattle to midges, and $\mathcal{R}_{m c}$ is the basic reproduction number of the infection from midges to cattle.

As the joint vector for two hosts, the midges are central for the infection. So let us take the midges' point of view. The product $\mathcal{R}_{m c} \mathcal{R}_{c m}$ is the average number of infectious midges produced via cattle hosts by a typical infectious midge when introduced at the disease-free equilibrium while $\mathcal{R}_{m s} \mathcal{R}_{s m}$ is the average number of infectious midges produced via sheep hosts. Combining both infection venues, $\mathcal{R}_{m c} \mathcal{R}_{c m}+\mathcal{R}_{m s} \mathcal{R}_{s m}$ is the average number of infectious midges produced via either host by one typical infectious midge. Since this reproduction requires two epidemiological generations, the basic reproduction number is given by

$$
\mathcal{R}_{0}=\left(\mathcal{R}_{m c} \mathcal{R}_{c m}+\mathcal{R}_{m s} \mathcal{R}_{s m}\right)^{1 / 2} .
$$

Theorem 3. Assume that the birth functions $b_{s}$ and $b_{m}$ satisfy (3.10) and (3.11) and are differentiable at $S_{s}^{0}$ and $S_{m}^{0}$, respectively, and that

$$
b_{s}^{\prime}\left(S_{s}^{0}\right)<\mu_{s}, \quad-\mu_{m} \leq e^{-\mu_{l} \tau} b_{m}^{\prime}\left(S_{m}^{0}\right)<\mu_{m} .
$$


Then, if $\mathcal{R}_{0}<1$, the disease free equilibrium

$$
\left(I_{c}, S_{s}, I_{s}, S_{m}, E_{m}, I_{m}\right)=\left(0, S_{s}^{0}, 0, S_{m}^{0}, 0,0\right)
$$

of system (2.2), (2.3), (2.8) is locally asymptotically stable.

Proof. The proof uses the principle of linearized stability for functional delay equations; see, for example, [26]. Since the $E_{m}$ equation may be dropped, it is sufficient to look at the linearized equations for the variables $S_{s}, S_{m}, I_{s}, I_{c}$ and $I_{m}$. From (2.2), (2.3) and (2.8), the linearized equations are

$$
\begin{aligned}
S_{s}^{\prime}(t) & =b_{s}^{\prime}\left(S_{s}^{0}\right) S_{s}(t)-\frac{\beta_{m s} S_{s}^{0}}{S_{s}^{0}+N_{c}} I_{m}(t)-\mu_{s} S_{s}(t), \\
S_{m}^{\prime}(t) & =e^{-\mu_{l} \tau} b_{m}^{\prime}\left(S_{m}^{0}\right) S_{m}(t-\tau)-\frac{\beta_{c m} S_{m}^{0} I_{c}(t)}{S_{s}^{0}+N_{c}}-\frac{\beta_{s m} S_{m}^{0} I_{s}(t)}{S_{s}^{0}+N_{c}}-\mu_{m} S_{m}(t), \\
I_{s}^{\prime}(t) & =\frac{\beta_{m s} S_{s}^{0}}{S_{s}^{0}+N_{c}} I_{m}(t)-\left(\gamma+\mu_{i s}\right) I_{s}(t) \\
I_{c}^{\prime}(t) & =\frac{\beta_{m c} S_{c}^{0}}{S_{s}^{0}+N_{c}} I_{m}(t)-\mu_{c} I_{c}(t), \\
I_{m}^{\prime}(t) & =\int_{0}^{\infty}\left(\frac{\beta_{c m} S_{m}^{0} I_{c}(t-\eta)}{S_{s}^{0}+N_{c}}+\frac{\beta_{s m} S_{m}^{0} I_{s}(t-\eta)}{S_{s}^{0}+N_{c}}\right) e^{-\mu_{e m} \eta} f(\eta) d \eta-\mu_{i m} I_{m}(t) .
\end{aligned}
$$

By [26], the disease-free equilibrium is locally asymptotically stable, if the origin is exponentially asymptotically stable for the linearized system. By [15, Sect. 3], this is the case if nontrivial solutions of the form $\left(S_{s}, S_{m}, I_{s}, I_{c}, I_{m}\right)=e^{\lambda t}\left(c_{1}, c_{2}, c_{3}, c_{4}, c_{5}\right)$ only exist if $\operatorname{Re} \lambda<0$.

Since the linearized system is reducible, nontrivial exponential solutions of this form can only exist when $\lambda$ satisfies one of the following characteristic equations,

$$
\begin{gathered}
\lambda=b_{s}^{\prime}\left(S_{s}^{0}\right)-\mu_{s}, \\
\lambda+\mu_{m}=e^{-\mu_{l} \tau} b_{m}^{\prime}\left(S_{m}^{0}\right) e^{-\lambda \tau}, \\
\lambda+\mu_{i m}=\frac{S_{m}^{0} \hat{f}\left(\lambda+\mu_{e m}\right)}{\left(S_{s}^{0}+N_{c}\right)^{2}}\left(\frac{\beta_{c m} \beta_{m c} N_{c}}{\lambda+\mu_{c}}+\frac{\beta_{s m} \beta_{m s} S_{s}^{0}}{\lambda+\gamma+\mu_{i s}}\right),
\end{gathered}
$$

where $\hat{f}$ is the Laplace transform of the kernel $f$, namely $\hat{f}(\lambda)=\int_{0}^{\infty} f(\eta) e^{-\lambda \eta} d \eta$. It is easy to see from our assumptions that any solution $\lambda$ of the first two characteristic equations has negative real part. Suppose, for contradiction, that a root $\lambda$ of (3.20) exists with $\operatorname{Re} \lambda \geq 0$. For such a $\lambda,\left|\lambda+\mu_{c}\right| \geq \mu_{c},\left|\lambda+\gamma+\mu_{i s}\right| \geq \gamma+\mu_{i s}$ and

$$
\left|\hat{f}\left(\lambda+\mu_{e m}\right)\right| \leq \int_{0}^{\infty} f(\eta) e^{-\left(\operatorname{Re} \lambda+\mu_{e m}\right) \eta} d \eta \leq \hat{f}\left(\mu_{e m}\right) .
$$

Therefore

$$
\left|\lambda+\mu_{i m}\right| \leq \frac{S_{m}^{0} \hat{f}\left(\mu_{e m}\right)}{\left(S_{s}^{0}+N_{c}\right)^{2}}\left(\frac{\beta_{c m} \beta_{m c} N_{c}}{\mu_{c}}+\frac{\beta_{s m} \beta_{m s} S_{s}^{0}}{\gamma+\mu_{i s}}\right)=\mathcal{R}_{0}^{2} \mu_{i m},
$$

which means that the root $\lambda$ lies in the disk in $\mathbf{C}$ centered at $-\mu_{i m}$ and of radius $\mathcal{R}_{0}^{2} \mu_{i m}$. This is a contradiction because, if $\mathcal{R}_{0}<1$, this disk is contained in the open left half of the complex plane and so $\lambda$ cannot satisfy $\operatorname{Re} \lambda \geq 0$. So, if $\mathcal{R}_{0}<1$, then all roots of (3.20) satisfy $\operatorname{Re} \lambda<0$ and local asymptotic stability holds.

We mention that (3.10) and (3.11) and monotone increasing $b_{m}$ imply (3.17) with strict inequalities being replaced by weak inequalities. 
3.3. Uniform weak persistence of the bluetongue. In this section we prove uniform weak persistence of the bluetongue in the midges if the basic reproduction number defined in (3.16) satisfies $\mathcal{R}_{0}>1$. Recall that the disease-free equilibrium is locally asymptotically stable if $\mathcal{R}_{0}<1$.

We prove that, under certain conditions, the variable representing the infectious midge compartment is persistent in the weak, uniform sense. In other words, for midges, there exists some constant $\epsilon>0$, which is independent of the initial conditions, such that $\lim \sup _{t \rightarrow \infty} I_{m}(t)>\epsilon$ provided that the disease is present at some point in time and that sheep are also present. We now introduce the basic reproduction numbers $\mathcal{R}_{0}^{\mathrm{s}}$ for sheep and $\mathcal{R}_{0}^{\mathrm{m}}$ for midges, given by

$$
\mathcal{R}_{0}^{s}=\frac{1}{\mu_{s}} \liminf _{S \rightarrow 0+} \frac{b_{s}(S)}{S}
$$

and

$$
\mathcal{R}_{0}^{m}=\frac{e^{-\mu_{l} \tau}}{\mu_{m}} \liminf _{S \rightarrow 0+} \frac{b_{m}(S)}{S} .
$$

For the midges, $\liminf _{S \rightarrow 0+} \frac{b_{m}(S)}{S}$ is the per capita oviposition rate at almost zero midge density, $e^{-\mu_{l} \tau}$ the probability of surviving the larval stage and $1 / \mu_{m}$ the mean life expectancy at the very end of the larval stage (i.e. the mean duration of the adult stage).

Theorem 4. Assume that the birth functions satisfy (3.10) and (3.11) and that $b_{m}$ is monotone increasing on $\left[0, S_{m}^{0}\right]$. If $\mathcal{R}_{0}>1, \mathcal{R}_{0}^{\mathrm{m}}>1$ and $\mathcal{R}_{0}^{\mathrm{s}}>1$, then there exists some $\epsilon>0$ such that

$$
\limsup _{t \rightarrow \infty} I_{m}(t)>\epsilon
$$

for all solutions with $S_{s}(0)>0, I_{m}(0)>0$, and $S_{m}(t)>0$ for some $t \in[-\tau, 0]$.

Proof. Suppose the statement is not true. Then, for $\epsilon>0$ to be chosen later, there exists a solution such that $I_{m}(0)>0$ and

$$
I_{m}^{\infty}=\limsup _{t \rightarrow \infty} I_{m}(t)<\epsilon .
$$

By Proposition 3.1, $I_{m}(t)>0$ for all $t \geq 0$. By Proposition 3.4, there exists some $c>0$ such that

$$
S_{c}(t)+S_{s}(t)+S_{m}(t) \leq N_{c}+N_{s}(t)+N_{m}(t) \leq c .
$$

The fluctuation method ([16], [28, Sec.A.3]) applied to (2.2) provides a sequence $\left(t_{j}\right)$ with $t_{j} \rightarrow \infty, I_{c}\left(t_{j}\right) \rightarrow I_{c}^{\infty}, I_{c}^{\prime}\left(t_{j}\right) \rightarrow 0$ as $j \rightarrow \infty$. By $(2.2)$,

$$
0 \leq \limsup _{j \rightarrow \infty} \frac{\beta_{m c} c I_{m}\left(t_{j}\right)}{N_{c}}-\mu_{c} I_{c}^{\infty} \leq \frac{\beta_{m c} c I_{m}^{\infty}}{N_{c}}-\mu_{c} I_{c}^{\infty} .
$$

This implies that $I_{c}^{\infty}<c_{1} \epsilon$ with a constant $c_{1}>0$ not depending on $\epsilon$. Similarly there is a constant $c_{2}>0$ such that $I_{s}^{\infty}<c_{2} \epsilon$.

Since $S_{m}(t)>0$ for some $t \in[-\tau, 0], S_{m}(t)>0$ for all $t>\tau$. Also $S_{m \infty} \geq$ $\lim _{n \rightarrow \infty} \inf _{[\tau+1, n]} S_{m}$. For $n \geq \tau+1$, choose $t_{n} \in[\tau+1, n]$ such that $S_{m}\left(t_{n}\right)=$ $\inf _{[\tau+1, n]} S_{m}$. Suppose that $S_{m \infty}=0$. Then $t_{n} \rightarrow \infty$ as $n \rightarrow \infty, S_{m}\left(t_{n}\right) \rightarrow 0$, $S_{m}^{\prime}\left(t_{n}\right) \leq 0$ and $S_{m}\left(t_{n}\right) \leq S_{m}\left(t_{n}-\tau\right)$ if $n$ is sufficiently large. It follows from the equation for $S_{m}$ in (2.8) that there is some $\nu>0$ such that $e^{\nu t} S_{m}(t)$ is an increasing function of $t$. This implies that $S_{m}\left(t_{n}-\tau\right) \leq e^{\nu \tau} S_{m}\left(t_{n}\right)$ and so $S_{m}\left(t_{n}-\tau\right) \rightarrow 0$ as 
$n \rightarrow \infty$. By (3.11) and $\mathcal{R}_{0}^{m}>1$, there exists some $\zeta>0$ such that $e^{-\mu_{l} \tau} b_{m}(S)>$ $\left(\mu_{m}+\zeta\right) S$ for all $S \in\left(0, S_{m}^{0} / 2\right]$. For sufficiently large $n$,

$S_{m}^{\prime}\left(t_{n}\right) \geq\left(\mu_{m}+\zeta\right) S_{m}\left(t_{n}-\tau\right)-\mu_{m} S_{m}\left(t_{n}\right)-\frac{\beta_{c m} I_{c}\left(t_{n}\right)}{N_{c}} S_{m}\left(t_{n}\right)-\frac{\beta_{s m} I_{s}\left(t_{n}\right)}{N_{c}} S_{m}\left(t_{n}\right)$.

For sufficiently large $n, S_{m}\left(t_{n}-\tau\right) \geq S_{m}\left(t_{n}\right), I_{c}\left(t_{n}\right) \leq c_{1} \epsilon$ and $I_{s}\left(t_{n}\right) \leq c_{2} \epsilon$, so

$$
S_{m}^{\prime}\left(t_{n}\right) \geq\left(\zeta-\left(\epsilon / N_{c}\right)\left[c_{1} \beta_{c m}+c_{2} \beta_{s m}\right]\right) S_{m}\left(t_{n}\right) .
$$

Note that $\zeta$ does not depend on $\epsilon$ as it is a property of the function $b_{m}$ and not the solution. If $\epsilon>0$ has been chosen small enough, $S_{m}^{\prime}\left(t_{n}\right)>0$, a contradiction. This implies that $S_{m \infty}>0$.

The fluctuation method applied to the first equation in (2.8) provides a sequence $\left(t_{j}\right)$ with $t_{j} \rightarrow \infty, S_{m}\left(t_{j}\right) \rightarrow S_{m \infty}$ and $S_{m}^{\prime}\left(t_{j}\right) \rightarrow 0$ as $j \rightarrow \infty$. Here $S_{m \infty}=$ $\liminf _{t \rightarrow \infty} S_{m}(t)$. By $(2.8)$,

$$
\begin{aligned}
0 \geq & e^{-\mu_{l} \tau} \liminf _{j \rightarrow \infty} b_{m}\left(S_{m}\left(t_{j}-\tau\right)\right)-\mu_{m} S_{m \infty} \\
& -\left[\limsup _{j \rightarrow \infty} \frac{\beta_{c m} I_{c}\left(t_{j}\right)}{N_{c}}+\limsup _{j \rightarrow \infty} \frac{\beta_{s m} I_{s}\left(t_{j}\right)}{N_{c}}\right] S_{m \infty} .
\end{aligned}
$$

Since $b_{m}$ is increasing on $\left[0, S_{m}^{0}\right], S_{m}^{\infty} \leq S_{m}^{0}$ by Proposition 3.4 and the subsequent remark. Hence

$$
\liminf _{j \rightarrow \infty} b_{m}\left(S_{m}\left(t_{j}-\tau\right)\right)=b_{m}(S) \quad \text { where } \quad S \in\left[S_{m \infty}, S_{m}^{\infty}\right] \subseteq\left(0, S_{m}^{0}\right] .
$$

Again, since $b_{m}$ is increasing on $\left[0, S_{m}^{\infty}\right]$,

$$
\liminf _{j \rightarrow \infty} b_{m}\left(S_{m}\left(t_{j}-\tau\right)\right) \geq b_{m}\left(S_{m \infty}\right) .
$$

Substituting this into a previous inequality,

$$
0 \geq e^{-\mu_{l} \tau} b_{m}\left(S_{m \infty}\right)-\mu_{m} S_{m \infty}-\frac{\beta_{c m} I_{c}^{\infty}}{N_{c}} S_{m \infty}-\frac{\beta_{s m} I_{s}^{\infty}}{N_{c}} S_{m \infty}
$$

Using the previous estimates, this gives

$$
0 \geq e^{-\mu_{l} \tau} b_{m}\left(S_{m \infty}\right)-\mu_{m} S_{m \infty}-\epsilon\left(\frac{\beta_{c m} c_{1}}{N_{c}}+\frac{\beta_{s m} c_{2}}{N_{c}}\right) S_{m \infty}
$$

Let $\delta>0$. Since $S_{m \infty}>0$, by (3.11) and (3.25), if $\epsilon>0$ is chosen small enough,

$$
S_{m \infty}>S_{m}^{\circ}-\delta
$$

Similar considerations, and further smallness constraints on $\epsilon$, provide $S_{m}^{\infty}<S_{m}^{\circ}+\delta$ and $S_{s}^{\infty}<S_{s}^{\circ}+\delta$.

From the differential equation for $S_{s}$ in $(2.3)$,

$$
S_{s}^{\prime}(t) \geq b_{s}\left(S_{s}(t)\right)-M \epsilon S_{s}(t)-\mu_{s} S_{s}(t)
$$

for $t>t_{\epsilon}$, with some constant $M>0$. Let $\delta \in\left(0, S_{s}^{0}\right)$. By the assumption $\mathcal{R}_{0}^{\mathrm{s}}>1$ and (3.10), there exists some $\zeta>0$ such that $b_{s}(S) \geq\left(\mu_{s}+\zeta\right) S$ for all $S \in\left[0, S_{s}^{0}-\delta\right]$. Choose $\epsilon>0$ so small that $M \epsilon<\zeta$. Then $S_{s}(t)$ satisfies $S_{s}^{\prime}(t) \geq(\zeta-M \epsilon) S_{s}(t)$ for as long as $t \geq t_{\epsilon}$ and $S_{s}(t) \leq S_{s}^{0}-\delta$, and so $S_{s}(t)$ grows at least exponentially while $S_{s}(t) \leq S_{s}^{0}-\delta$. But this implies that $S_{s}(t) \geq S_{s}^{0}-\delta$ for sufficiently large $t>0$. 
After shifting forward in time, it can be assumed that

$0<I_{m}(t) \leq \epsilon, \quad I_{c}(t) \leq c_{1} \epsilon, \quad I_{s}(t) \leq c_{2} \epsilon$,

$0<S_{m}^{\circ}-\delta \leq S_{m}(t) \leq S_{m}^{\circ}+\delta, \quad 0<S_{s}^{\circ}-\delta \leq S_{s}(t) \leq S_{s}^{\circ}+\delta, \quad 0 \leq S_{c}^{\circ}-\delta \leq S_{c}(t)$

for all $t \geq 0$, where the last inequality follows from $S_{c}(t)=N_{c}-I_{c}(t)=S_{c}^{0}-I_{c}(t) \geq$ $S_{c}^{0}-c_{1} \epsilon \geq S_{c}^{0}-\delta$ for sufficiently small $\epsilon$. These give the following differential inequalities,

$$
\begin{aligned}
I_{c}^{\prime}(t) \geq & \frac{\beta_{m c}\left(S_{c}^{\circ}-\delta\right) I_{m}(t)}{c_{2} \epsilon+S_{s}^{\circ}+\delta+N_{c}}-\mu_{c} I_{c}(t) \\
I_{s}^{\prime}(t) \geq & \frac{\beta_{m s}\left(S_{s}^{\circ}-\delta\right) I_{m}(t)}{c_{2} \epsilon+S_{s}^{\circ}+\delta+N_{c}}-\gamma I_{s}(t)-\mu_{i s} I_{s}(t), \\
I_{m}^{\prime}(t) \geq & \int_{0}^{t}\left(\frac{\beta_{c m}\left(S_{m}^{\circ}-\delta\right) I_{c}(r)}{c_{2} \epsilon+S_{s}^{\circ}+\delta+N_{c}}+\frac{\beta_{s m}\left(S_{m}^{\circ}-\delta\right) I_{s}(r)}{c_{2} \epsilon+S_{s}^{\circ}+\delta+N_{c}}\right) e^{-\mu_{e m}(t-r)} f(t-r) d r \\
& -\mu_{i m} I_{m}(t)
\end{aligned}
$$

where we have replaced $-\infty$ by 0 in the lower limit of integration, since the contribution from $\int_{-\infty}^{0}$ is non-negative. Applying the Laplace transform to the differential inequalities and using the usual rules, for example, that the Laplace transform turns convolutions into products, and the fact that $I_{c}(0), I_{s}(0), I_{m}(0) \geq 0$,

$$
\begin{aligned}
\lambda \hat{I}_{c}(\lambda) & \geq \frac{\beta_{m c}\left(S_{c}^{\circ}-\delta\right)}{c_{2} \epsilon+S_{s}^{\circ}+\delta+N_{c}} \hat{I}_{m}(\lambda)-\mu_{c} \hat{I}_{c}(\lambda), \\
\lambda \hat{I}_{s}(\lambda) & \geq \frac{\beta_{m s}\left(S_{s}^{\circ}-\delta\right)}{c_{2} \epsilon+S_{s}^{\circ}+\delta+N_{c}} \hat{I}_{m}(\lambda)-\gamma \hat{I}_{s}(\lambda)-\mu_{i s} \hat{I}_{s}(\lambda), \\
\lambda \hat{I}_{m}(\lambda) & \geq \frac{S_{m}^{\circ}-\delta}{c_{2} \epsilon+S_{s}^{\circ}+\delta+N_{c}}\left(\beta_{c m} \hat{I}_{c}(\lambda)+\beta_{s m} \hat{I}_{s}(\lambda)\right) \hat{f}\left(\mu_{e m}+\lambda\right)-\mu_{i m} \hat{I}_{m}(\lambda),
\end{aligned}
$$

where $\lambda$ is the transform variable, taken as real and strictly positive. Reorganizing the terms gives

$$
\begin{aligned}
\hat{I}_{c}(\lambda) & \geq \frac{1}{\left(\lambda+\mu_{c}\right)} \frac{\beta_{m c}\left(S_{c}^{\circ}-\delta\right)}{\left(c_{2} \epsilon+S_{s}^{\circ}+\delta+N_{c}\right)} \hat{I}_{m}(\lambda), \\
\hat{I}_{s}(\lambda) & \geq \frac{1}{\left(\lambda+\gamma+\mu_{i s}\right)} \frac{\beta_{m s}\left(S_{s}^{\circ}-\delta\right)}{\left(c_{2} \epsilon+S_{s}^{\circ}+\delta+N_{c}\right)} \hat{I}_{m}(\lambda), \\
\hat{I}_{m}(\lambda) & \geq \frac{1}{\lambda+\mu_{i m}}\left(\frac{\beta_{c m}\left(S_{m}^{\circ}-\delta\right)}{c_{2} \epsilon+S_{s}^{\circ}+\delta+N_{c}} \hat{I}_{c}(\lambda)+\frac{\beta_{s m}\left(S_{m}^{\circ}-\delta\right)}{c_{2} \epsilon+S_{s}^{\circ}+\delta+N_{c}} \hat{I}_{s}(\lambda)\right) \hat{f}\left(\mu_{e m}+\lambda\right) .
\end{aligned}
$$

Substituting the first and second inequality into the third and dividing by $\hat{I}_{m}(\lambda)>0$,

$$
\begin{aligned}
1 \geq & \frac{1}{\lambda+\mu_{i m}} \hat{f}\left(\mu_{e m}+\lambda\right)\left[\frac{\beta_{c m}\left(S_{m}^{\circ}-\delta\right)}{\left(c_{2} \epsilon+S_{s}^{\circ}+\delta+N_{c}\right)} \frac{1}{\left(\lambda+\mu_{c}\right)} \frac{\beta_{m c}\left(S_{c}^{\circ}-\delta\right)}{\left(c_{2} \epsilon+S_{s}^{\circ}+\delta+N_{c}\right)}\right. \\
& \left.+\frac{\beta_{s m}\left(S_{m}^{\circ}-\delta\right)}{\left(c_{2} \epsilon+S_{s}^{\circ}+\delta+N_{c}\right)} \frac{1}{\left(\lambda+\gamma+\mu_{i s}\right)} \frac{\beta_{m s}\left(S_{s}^{\circ}-\delta\right)}{\left(c_{2} \epsilon+S_{s}^{\circ}+\delta+N_{c}\right)}\right] .
\end{aligned}
$$

This inequality holds for all $\lambda>0$. Letting $\lambda \rightarrow 0$, then $\epsilon \rightarrow 0$ and finally $\delta \rightarrow 0$, and recalling that $S_{c}^{\circ}=N_{c}$, gives

$$
1 \geq\left[\frac{\beta_{c m} S_{m}^{\circ}}{\left(S_{s}^{\circ}+N_{c}\right)} \frac{1}{\mu_{c}} \frac{\beta_{m c} S_{c}^{\circ}}{\left(S_{s}^{\circ}+N_{c}\right)}+\frac{\beta_{s m} S_{m}^{\circ}}{\left(S_{s}^{\circ}+N_{c}\right)} \frac{1}{\left(\gamma+\mu_{i s}\right)} \frac{\beta_{m s} S_{s}^{\circ}}{\left(S_{s}^{\circ}+N_{c}\right)}\right] \frac{1}{\mu_{i m}} \hat{f}\left(\mu_{e m}\right)=\mathcal{R}_{0}^{2} .
$$

This contradicts the assumption $\mathcal{R}_{0}>1$.

Note that the Laplace transform has previously been used to establish uniform weak persistence; see [28, Sec.22.3] and [21]. 
3.4. Global stability of the disease free equilibria. In this section we obtain some conditions that are sufficient for global stability of the disease free equilibria $\left(I_{c}, S_{s}, I_{s}, S_{m}, E_{m}, I_{m}\right)=\left(0, S_{s}^{0}, 0, S_{m}^{0}, 0,0\right)$ and $\left(0,0,0, S_{m}^{0}, 0,0\right)$. The conditions are stronger than those of Theorem 3.

Theorem 5. Suppose that the birth function $b_{s}\left(S_{s}\right)$ satisfies (3.10) and is monotone non-decreasing on $\left[0, S_{s}^{0}\right]$, and that the birth function $b_{m}\left(S_{m}\right)$ satisfies (3.11) and is monotone non-decreasing on $\left[0, S_{m}^{0}\right]$. Assume also that

$$
\frac{S_{m}^{0}}{N_{c}}\left(\frac{\beta_{c m} \beta_{m c}}{\mu_{c} \mu_{i m}}+\frac{\beta_{s m}}{\gamma+\mu_{i s}} \mathcal{R}_{m s}\right) \hat{f}\left(\mu_{e m}\right)<1 .
$$

Then $I_{m}(t), I_{c}(t), I_{s}(t) \rightarrow 0$ as $t \rightarrow \infty$. Moreover:

(i) if $\mathcal{R}_{0}^{m}>1$ and $\mathcal{R}_{0}^{s}>1$, the disease free equilibrium $\left(I_{c}, S_{s}, I_{s}, S_{m}, E_{m}, I_{m}\right)=$ $\left(0, S_{s}^{0}, 0, S_{m}^{0}, 0,0\right)$ of (2.2), (2.3) and (2.8) attracts all solutions whose initial data satisfy (3.1) and the additional requirements that $S_{s}(0)+I_{s}(0)>0$ and $S_{m 0}(\theta) \not \equiv 0$ on $[-\tau, 0]$;

(ii) if $\mathcal{R}_{0}^{m}>1$, the disease free equilibrium $\left(I_{c}, S_{s}, I_{s}, S_{m}, E_{m}, I_{m}\right)=\left(0,0,0, S_{m}^{0}, 0,0\right)$ of (2.2), (2.3) and (2.8) attracts all solutions whose initial data satisfy (3.1) and the additional requirements that $S_{s}(0)=0=I_{s}(0)$ and $S_{m 0}(\theta) \not \equiv 0$ on $[-\tau, 0]$.

Proof. By Proposition 3.4,

$$
S_{m}^{\infty}:=\limsup _{t \rightarrow \infty} S_{m}(t) \leq S_{m}^{0}, \quad S_{s}^{\infty}:=\limsup _{t \rightarrow \infty} S_{s}(t) \leq \limsup _{t \rightarrow \infty} N_{s}(t) \leq S_{s}^{0} .
$$

We apply the fluctuation method to the $I_{s}$ equation in (2.3); actually to the following inequality,

$$
I_{s}^{\prime}(t) \leq \frac{\beta_{m s} S_{s}(t) I_{m}(t)}{S_{s}(t)+N_{c}}-\left(\gamma+\mu_{i s}\right) I_{s}(t)
$$

By [28, Prop.A.22] there exists a sequence $\left(t_{j}\right)$ such that

$$
t_{j} \rightarrow \infty, \quad I_{s}\left(t_{j}\right) \rightarrow I_{s}^{\infty}:=\limsup _{t \rightarrow \infty} I_{s}(t), \quad I_{s}^{\prime}\left(t_{j}\right) \rightarrow 0, \quad j \rightarrow \infty .
$$

Since $x /\left(x+N_{c}\right)$ is an increasing function of $x$,

$$
0 \leq \frac{\beta_{m s} S_{s}^{\infty} I_{m}^{\infty}}{S_{s}^{\infty}+N_{c}}-\left(\gamma+\mu_{i s}\right) I_{s}^{\infty} .
$$

Solving for $I_{s}^{\infty}$ and using $S_{s}^{\infty} \leq S_{s}^{0}$,

$$
I_{s}^{\infty} \leq \frac{\beta_{m s} S_{s}^{0} I_{m}^{\infty}}{\left(\gamma+\mu_{i s}\right)\left(S_{s}^{0}+N_{c}\right)}=: \phi\left(I_{m}^{\infty}\right) .
$$

Similarly, from the infectious cattle equation $(2.2)$, since $S_{c}(t) \leq N_{c}$,

$$
I_{c}^{\prime}(t) \leq \frac{\beta_{m c} N_{c} I_{m}(t)}{I_{s}(t)+S_{s}(t)+N_{c}}-\mu_{c} I_{c}(t) \leq \beta_{m c} I_{m}(t)-\mu_{c} I_{c}(t) .
$$

The fluctuation method yields

$$
I_{c}^{\infty} \leq \frac{\beta_{m c}}{\mu_{c}} I_{m}^{\infty} .
$$

For the infectious midges equation the situation is more delicate because of the delay. By non-negativity of solution components, the function $B(t)$ defined by $(2.7)$ satisfies

$$
B(t) \leq\left(\frac{\beta_{c m} I_{c}(t)}{N_{c}}+\frac{\beta_{s m} I_{s}(t)}{I_{s}(t)+N_{c}}\right) S_{m}(t)
$$


We apply the fluctuation method to the equation

$$
I_{m}^{\prime}(t)=-\mu_{i m} I_{m}(t)+\int_{0}^{\infty} B(t-\eta) e^{-\mu_{e m} \eta} f(\eta) d \eta .
$$

By [28, Prop.A.22], there exists a sequence $\left(t_{j}\right)$ such that

$$
t_{j} \rightarrow \infty, \quad I_{m}\left(t_{j}\right) \rightarrow I_{m}^{\infty}, \quad I_{m}^{\prime}\left(t_{j}\right) \rightarrow 0, \quad j \rightarrow \infty .
$$

Using this and Fatou's lemma [28, p. 468],

$$
\begin{aligned}
0 & \leq-\mu_{i m} I_{m}^{\infty}+\int_{0}^{\infty} \limsup _{j \rightarrow \infty} B\left(t_{j}-\eta\right) e^{-\mu_{e m} \eta} f(\eta) d \eta \\
& \leq-\mu_{i m} I_{m}^{\infty}+\limsup _{t \rightarrow \infty} B(t) \int_{0}^{\infty} e^{-\mu_{e m} \eta} f(\eta) d \eta .
\end{aligned}
$$

Solving for $I_{m}^{\infty}$, using (3.32) and that $x /\left(x+N_{c}\right)$ increases with $x$,

$$
I_{m}^{\infty} \leq\left(\frac{\beta_{c m} I_{c}^{\infty}}{N_{c}}+\frac{\beta_{s m} I_{s}^{\infty}}{I_{s}^{\infty}+N_{c}}\right) S_{m}^{\infty} \frac{\hat{f}\left(\mu_{e m}\right)}{\mu_{i m}} .
$$

Suppose that $I_{m}^{\infty}>0$. Substituting $S_{m}^{\infty} \leq S_{m}^{0}$ and (3.31) into this inequality, making use of (3.29), and again using that $x /\left(x+N_{c}\right)$ is increasing in $x$, gives

$$
I_{m}^{\infty} \leq\left(\frac{\beta_{c m} \beta_{m c} I_{m}^{\infty}}{\mu_{c} N_{c}}+\frac{\beta_{s m} \phi\left(I_{m}^{\infty}\right)}{\phi\left(I_{m}^{\infty}\right)+N_{c}}\right) S_{m}^{0} \frac{\hat{f}\left(\mu_{e m}\right)}{\mu_{i m}}
$$

so that, dividing by $I_{m}^{\infty}>0$ and again using (3.29),

$$
1 \leq\left(\frac{\beta_{c m} \beta_{m c}}{\mu_{c} N_{c}}+\frac{\beta_{s m}}{\left(\phi\left(I_{m}^{\infty}\right)+N_{c}\right)} \frac{\beta_{m s} S_{s}^{0}}{\left(\gamma+\mu_{i s}\right)\left(S_{s}^{0}+N_{c}\right)}\right) S_{m}^{0} \frac{\hat{f}\left(\mu_{e m}\right)}{\mu_{i m}} .
$$

Since $\phi\left(I_{m}^{\infty}\right)>0$, this contradicts the threshold condition (3.26). Thus, under this condition, $I_{m}^{\infty}=0$ and also $I_{c}^{\infty}=0=I_{s}^{\infty}$ by (3.31) and (3.29). Hence $\left(I_{s}(t), I_{c}(t), I_{m}(t)\right) \rightarrow(0,0,0)$ as $t \rightarrow \infty$, and obviously $S_{c}(t) \rightarrow N_{c}$.

In this limit, the equations for the numbers of susceptible sheep and midges become (3.7) and (3.8). The former is just a one-dimensional ODE, and assumption (3.10) together with $S_{s}(0)+I_{s}(0)>0$ and $\gamma>0$ ensures that $S_{s} \rightarrow S_{s}^{0}$ if $\mathcal{R}_{0}^{s}>1$.

We consider the first equation in (2.8) as a scalar asymptotically autonomous differential-delay equation,

$$
S_{m}^{\prime}(t)=e^{-\mu_{l} \tau} b_{m}\left(S_{m}(t-\tau)\right)-\mu_{m} S_{m}(t)-S_{m}(t) \psi(t),
$$

where $\psi$ is a nonnegative differentiable function on $\mathbb{R}_{+}$and $\psi(t) \rightarrow 0$ as $t \rightarrow \infty$. Similarly as in the proof of Theorem 4, we first show that $S_{m \infty}=\liminf _{t \rightarrow \infty} S_{m}(t)>0$ and then

$$
0 \geq e^{-\mu_{l} \tau} b_{m}\left(S_{m \infty}\right)-\mu_{m} S_{m \infty} .
$$

By (3.11), $S_{m \infty} \geq S_{m}^{0}$. Since $S_{m}^{\infty} \leq S_{m}^{0}$ by Proposition 3.4, $S_{m}(t) \rightarrow S_{m}^{0}$ as $t \rightarrow \infty$ if $\mathcal{R}_{0}^{m}>1$.

REMARK. If $b_{s}$ and $b_{m}$ are not assumed to be monotone non-decreasing, then the attraction result above remains true if the threshold condition is replaced by

$$
\frac{\breve{S}_{m}}{N_{c}}\left(\frac{\beta_{c m} \beta_{m c}}{\mu_{c} \mu_{i m}}+\frac{\beta_{s m}}{\gamma+\mu_{i s}} \frac{1}{\mu_{i m}} \frac{\beta_{m s} \breve{S}_{s}}{\left(\breve{S}_{s}+N_{c}\right)}\right) \hat{f}\left(\mu_{e m}\right)<1,
$$

with $\breve{S}_{s}$ and $\breve{S}_{m}$ from Proposition 3.4. 
3.5. Uniform weak persistence of midges. Let $S_{m}^{\sharp}$ be defined by the equation

$$
\frac{S_{m}^{\sharp}}{N_{c}}\left(\frac{\beta_{c m} \beta_{m c}}{\mu_{c} \mu_{i m}}+\frac{\beta_{s m}}{\gamma+\mu_{i s}} \frac{1}{\mu_{i m}} \frac{\beta_{m s} \breve{S}_{s}}{\left(\breve{S}_{s}+N_{c}\right)}\right) \hat{f}\left(\mu_{e m}\right)=1,
$$

with $\breve{S}_{s}$ from Proposition 3.4.

Proposition 3.5. Assume that the birth functions satisfy (3.10) and (3.11) and that $b_{m}$ is monotone increasing. If $\mathcal{R}_{0}^{m}>1$, where $\mathcal{R}_{0}^{m}$ is defined by (3.23), then, for all solutions with $S_{m 0}(\theta) \not \equiv 0$ on $[-\tau, 0]$,

$$
\limsup _{t \rightarrow \infty} S_{m}(t) \geq \min \left\{S_{m}^{\sharp}, S_{m}^{0}\right\} .
$$

Proof. Suppose $\lim \sup _{t \rightarrow \infty} S_{m}(t)<\min \left\{S_{m}^{\sharp}, S_{m}^{0}\right\}$. The same proof as for Theorem 5 shows that $S_{m}(t) \rightarrow S_{m}^{0}$, a contradiction.

4. Exponentially distributed incubation period. Assuming that the incubation period is exponentially distributed with mean time $1 / \alpha$ in the exposed class, $f(\xi)=\alpha e^{-\alpha \xi}$ with $\alpha>0$. In this case, equation (2.5) becomes

$$
E_{m}(t)=\int_{-\infty}^{t} B(\eta) e^{-\left(\alpha+\mu_{e m}\right)(t-\eta)} d \eta
$$

Neglecting the pre-adult stages (i.e., setting $\tau=0$ ), using (2.8) and differentiating (4.1), the equations for the midges become the ordinary differential equation (ODE) system

$$
\begin{aligned}
S_{m}^{\prime}(t) & =b_{m}\left(S_{m}\right)-\mu_{m} S_{m}-\frac{\beta_{c m} S_{m} I_{c}}{I_{s}+S_{s}+N_{c}}-\frac{\beta_{s m} S_{m} I_{s}}{I_{s}+S_{s}+N_{c}}, \\
E_{m}^{\prime}(t) & =\frac{\beta_{c m} S_{m} I_{c}}{I_{s}+S_{s}+N_{c}}+\frac{\beta_{s m} S_{m} I_{s}}{I_{s}+S_{s}+N_{c}}-\left(\alpha+\mu_{e m}\right) E_{m}, \\
I_{m}^{\prime}(t) & =\alpha E_{m}-\mu_{i m} I_{m} .
\end{aligned}
$$

The complete model in this case is given by the six dimensional ODE system (2.2), (2.3), and (4.2) with $S_{c}=N_{c}-I_{c}$, and initial conditions $S_{s}(0), S_{m}(0)>0$, $I_{c}(0), I_{s}(0), I_{m}(0) \geq 0$ with $I_{c}(0)+I_{s}(0)+I_{m}(0)>0$ and $E(0)$ satisfying (4.1) with $t=0$.

4.1. Basic reproduction number revisited. As in the general model, there are four disease free equilibria, three of which are unstable: see Section 3.1.

We focus on the other disease free equilibrium with $S_{s}=S_{s}^{0}>0, S_{m}=S_{m}^{0}>0$ and satisfying $b_{s}\left(S_{s}^{0}\right)=\mu_{s} S_{s}^{0}$ and $b_{m}\left(S_{m}^{0}\right)=\mu_{m} S_{m}^{0}$, and all other variables equal to zero. Assumptions on the birth functions are made as in Section 3.1, namely (3.10) and (3.11). After observing that $\hat{f}\left(\mu_{e m}\right)=\frac{\alpha}{\alpha+\mu_{e m}}$, the expression for $\mathcal{R}_{0}$ can systematically be derived using the next generation matrix method $[9,29]$.

From Section 3.1, the system is stable in the absence of disease, thus it is sufficient to consider the four dimensional system in the infected variables $E_{m}, I_{m}, I_{s}, I_{c}$ linearized at the disease free equilibrium. Writing the Jacobian matrix $J=F-V$, where $F$ contains the new infections and $V$ contains the transfer between compart- 
ments gives

$$
\begin{aligned}
F=\frac{1}{S_{s}^{0}+N_{c}} & {\left[\begin{array}{cccc}
0 & 0 & \beta_{s m} S_{m}^{0} & \beta_{c m} S_{m}^{0} \\
0 & 0 & 0 & 0 \\
0 & \beta_{m s} S_{s}^{0} & 0 & 0 \\
0 & \beta_{m c} N_{c} & 0 & 0
\end{array}\right], } \\
V & =\left[\begin{array}{cccc}
\alpha+\mu_{e m} & 0 & 0 & 0 \\
-\alpha & \mu_{i m} & 0 & 0 \\
0 & 0 & \gamma+\mu_{i s} & 0 \\
0 & 0 & 0 & \mu_{c}
\end{array}\right] .
\end{aligned}
$$

After computing $F V^{-1}$, its spectral radius equals $\mathcal{R}_{0}$ defined in (3.16), the basic reproduction number for this special case, and Theorem 3 follows from [9, 29].

Our bluetongue model ODE system has similarities to the model for bluetongue spread in UK livestock developed by Gubbins et al. [11] with exponential distributions; see especially [11, equation (3.1)] for the basic reproduction number in their model. Hartemink et al. [13, page 155] also give a similar expression.

4.2. Type reproduction numbers. In order to consider control of bluetongue by either reducing infectious midges or exposed sheep, it is useful to consider type reproduction numbers $T_{\ell}$ as introduced by [14, 25]. Using the result of [25, Appendix A] taking infected individuals of type $\ell$ only, $T_{\ell}=r\left(M_{\ell}\right)$, with

$$
M_{\ell}=P_{\ell} K\left(I-\left(I-P_{\ell}\right) K\right)^{-1}
$$

where $K$ is the next generation matrix and $P_{\ell}$ is the projection matrix having 1 in the $\ell, \ell$ entry and 0 elsewhere. Note that in general, $T_{\ell}=1$ iff $\mathcal{R}_{0}=1$. Because $F$ in (4.3) has a zero row, $K$ is taken to be the $3 \times 3$ matrix on the infected variables $E_{m}, I_{s}, I_{c}$, namely

$$
K=\frac{1}{S_{s}^{0}+N_{c}}\left[\begin{array}{ccc}
0 & \frac{\beta_{s m} S_{m}^{0}}{\gamma+\mu_{i s}} & \frac{\beta_{c m} S_{m}^{0}}{\mu_{c}} \\
\frac{\alpha \beta_{m s} S_{s}^{0}}{\mu_{i m}\left(\alpha+\mu_{e m}\right)} & 0 & 0 \\
\frac{\alpha \beta_{m c} N_{c}}{\mu_{i m}\left(\alpha+\mu_{e m}\right)} & 0 & 0
\end{array}\right] .
$$

Assume that one exposed midge is introduced into a susceptible population of midges, sheep and cattle, thus $\ell=1$ and $P_{1}=\operatorname{diag}(1,0,0)$. From (4.4) inserting (4.5), gives $T_{1}=\mathcal{R}_{0}^{2}$ with $\mathcal{R}_{0}$ given by (3.16). Thus the expected number of secondary exposed midges that result from the introduction of a single exposed midge is $\mathcal{R}_{0}^{2}$, as infection from midge to midge requires two generations. If culling of midges is a strategy under consideration, then a proportion $1-1 / \mathcal{R}_{0}^{2}$ should be culled to eliminate infection.

By contrast, assume that one infectious sheep is introduced into a susceptible population, thus $\ell=2$ and $P_{2}=\operatorname{diag}(0,1,0)$. From (4.4) using (4.5) gives $T_{2}=$ $\mathcal{R}_{m s} \mathcal{R}_{s m} /\left(1-\mathcal{R}_{m c} \mathcal{R}_{c m}\right)$, in which the various reproduction numbers are given by (3.12) - (3.15), and it is assumed that $\mathcal{R}_{m c} \mathcal{R}_{c m}<1$, i.e., the disease cannot persist in the midges and cattle alone. If it is desired to treat only the sheep, perhaps by vaccination, to eradicate the disease, then a proportion $1-1 / T_{2}$ must be vaccinated.

Similarly, assuming that one infectious cow is introduced, the type reproduction number is $T_{3}=\mathcal{R}_{m c} \mathcal{R}_{c m} /\left(1-\mathcal{R}_{m s} \mathcal{R}_{s m}\right)$, where it is assumed that the disease cannot persist in the midges and sheep alone. Treating only the cattle, a fraction $1-1 / T_{3}$ must be treated to eradicate the disease.

4.3. Backward bifurcation. Huang et al. [18] first described backward bifurcation in a multiple-group HIV/AIDS model, and we demonstrate the possibility of this phenomenon in our bluetongue model. Consider a simplified model in which the 
birth functions are constant, there are no cattle, no sheep recover, and the midges have no incubation period and do not die from the disease, i.e., $b_{m}\left(S_{m}\right)=b_{m}$, $b_{s}\left(S_{s}\right)=b_{s}, N_{c}=0, \gamma=0, \alpha \rightarrow \infty$ and $\delta_{m}=0$. The system then becomes

$$
\begin{aligned}
S_{m}^{\prime}(t) & =b_{m}-\mu_{m} S_{m}-\frac{\beta_{s m} S_{m} I_{s}}{I_{s}+S_{s}} \\
I_{m}^{\prime}(t) & =\frac{\beta_{s m} S_{m} I_{s}}{I_{s}+S_{s}}-\mu_{m} I_{m} \\
S_{s}^{\prime}(t) & =b_{s}-\frac{\beta_{m s} S_{s} I_{m}}{I_{s}+S_{s}}-\mu_{s} S_{s} \\
I_{s}^{\prime}(t) & =\frac{\beta_{m s} S_{s} I_{m}}{I_{s}+S_{s}}-\mu_{i s} I_{s}
\end{aligned}
$$

where $\mu_{i s}=\mu_{s}+\delta_{s}$, with non-negative initial data and $I_{m}(0)+I_{s}(0)>0$. This four dimensional model is equivalent to the one considered by Bowman et al. [5] and further analyzed by Jiang et al. [19] for the transmission of West Nile virus in the primary mosquito-bird cycle. The disease free equilibrium always exists and is given by $\left(S_{m}, I_{m}, S_{s}, I_{s}\right)=\left(b_{m} / \mu_{m}, 0, b_{s} / \mu_{s}, 0\right)$. Note that the constant birth functions taken here do not satisfy all the conditions of (3.10), (3.11), but as we only consider this simplified model near the DFE, this causes no problem. From (3.16) the basic reproduction number for this simplified model is

$$
\mathcal{R}_{0}=\left\{\frac{b_{m} \mu_{s} \beta_{s m} \beta_{m s}}{b_{s} \mu_{m}^{2} \mu_{i s}}\right\}^{\frac{1}{2}}=\left(\mathcal{R}_{m s} \mathcal{R}_{s m}\right)^{1 / 2}
$$

If $\mathcal{R}_{0}>1$, then there is a unique endemic equilibrium that is locally asymptotically stable [19, Theorem 3.3]. If $\mathcal{R}_{0}<1$, then the disease free equilibrium is locally asymptotically stable, but a backward bifurcation may occur if $\delta_{s}>\mu_{s}$, i.e. the sheep disease induced death rate is greater than the natural death rate: details are given in [19, Section 4]. By continuity with respect to parameters, our six dimensional ODE bluetongue model also has the possibility of backward bifurcation, in which case $\mathcal{R}_{0}$ is not sufficient to determine whether the disease dies out, as solutions are initial value dependent for a range of $\mathcal{R}_{0}$ values below one.

4.4. A stable sheep-free disease equilibrium. To demonstrate the possibility that the disease wipes out the sheep population, we consider a simplified ODE model without an incubation period and a juvenile stage for the midges, namely

$$
\begin{aligned}
I_{c}^{\prime} & =\frac{\beta_{m c}\left(N_{c}-I_{c}\right) I_{m}}{I_{s}+S_{s}+N_{c}}-\mu_{c} I_{c} \\
S_{m}^{\prime} & =b_{m}\left(S_{m}\right)-\mu_{m} S_{m}-\frac{\beta_{c m} S_{m} I_{c}}{I_{s}+S_{s}+N_{c}}-\frac{\beta_{s m} S_{m} I_{s}}{I_{s}+S_{s}+N_{c}}, \\
I_{m}^{\prime} & =\frac{\beta_{c m} S_{m} I_{c}}{I_{s}+S_{s}+N_{c}}+\frac{\beta_{s m} S_{m} I_{s}}{I_{s}+S_{s}+N_{c}}-\mu_{i m} I_{m}, \\
S_{s}^{\prime} & =b_{s}\left(S_{s}\right)-\frac{\beta_{m s} S_{s} I_{m}}{I_{s}+S_{s}+N_{c}}+\gamma I_{s}-\mu_{s} S_{s}, \\
I_{s}^{\prime} & =\frac{\beta_{m s} S_{s} I_{m}}{I_{s}+S_{s}+N_{c}}-\gamma I_{s}-\mu_{i s} I_{s} .
\end{aligned}
$$


4.4.1. The equilibrium. Denoting the steady state variables with a superscript $\sim$ and setting $S_{s}=I_{s}=0$ gives

$$
\begin{aligned}
& 0=\frac{\beta_{m c}\left(N_{c}-\tilde{I}_{c}\right) \tilde{I}_{m}}{N_{c}}-\mu_{c} \tilde{I}_{c}, \\
& 0=b_{m}\left(\tilde{S}_{m}\right)-\mu_{m} \tilde{S}_{m}-\frac{\beta_{c m} \tilde{S}_{m} \tilde{I}_{c}}{N_{c}}, \\
& 0=\frac{\beta_{c m} \tilde{S}_{m} \tilde{I}_{c}}{N_{c}}-\mu_{i m} \tilde{I}_{m} .
\end{aligned}
$$

From the first and third equations with $\tilde{I}_{c}>0$,

$$
0=\frac{\beta_{m c}\left(N_{c}-\tilde{I}_{c}\right)}{N_{c}} \frac{\beta_{c m} \tilde{S}_{m}}{N_{c}}-\mu_{c} \mu_{i m}
$$

We rewrite this equation as

$$
\frac{\tilde{I}_{c}}{N_{c}}=1-\frac{1}{\tilde{\mathcal{R}}_{0}^{2}} \frac{S_{m}^{0}}{\tilde{S}_{m}}
$$

with

$$
\tilde{\mathcal{R}}_{0}=\sqrt{\frac{\beta_{m c}}{\mu_{i m}} \frac{\beta_{c m} S_{m}^{0}}{\mu_{c} N_{c}}} .
$$

Similarly to Section $3.2, \tilde{\mathcal{R}}_{0}$ can be interpreted as the basic disease reproduction number of the cattle-midge bluetongue system (without the sheep). If there is a positive equilibrium, it follows from the second equation in (4.9) that $\tilde{S}_{m}<S_{m}^{0}$ and from (4.11) that $\tilde{\mathcal{R}}_{0}>1$. We divide the second equation in (4.9) by $\tilde{S}_{m}$ and substitute (4.11) to obtain

$$
0=\frac{b_{m}\left(\tilde{S}_{m}\right)}{\tilde{S}_{m}}-\mu_{m}-\beta_{c m}\left(1-\frac{1}{\tilde{\mathcal{R}}_{0}^{2}} \frac{S_{m}^{0}}{\tilde{S}_{m}}\right)
$$

Assume that $\tilde{\mathcal{R}}_{0}>1$. By (3.11), the right hand side of this equation is negative for $\tilde{S}_{m}=S_{m}^{0}$ and positive for $\tilde{S}_{m}=S_{m}^{0} / \tilde{\mathcal{R}}_{0}^{2}$. By the intermediate value theorem, there exists a solution $\tilde{S}_{m}$ between $S_{m}^{0} / \tilde{\mathcal{R}}_{0}^{2}$ and $S_{m}^{0}$.

The solution is unique if it is assumed that the per capita midge reproduction rate $b_{m}(x) / x$ is a decreasing function of midge density $x$.

4.4.2. The linearization about the equilibrium. The Jacobian matrix taken at this sheep-free disease equilibrium is of block form and its eigenvalues are eigenvalues of the matrix

$$
J=\left[\begin{array}{ccc}
-\frac{\beta_{m c} \tilde{I}_{m}}{N_{c}}-\mu_{c} & 0 & \beta_{m c} \frac{N_{c}-\tilde{I}_{c}}{N_{c}} \\
-\frac{\beta_{c m} \tilde{S}_{m}}{N_{c}} & -\kappa & 0 \\
\frac{\beta_{c c} \tilde{S}_{m}}{N_{c}} & \frac{\beta_{c m} \tilde{I}_{c}}{N_{c}} & -\mu_{i m}
\end{array}\right]
$$

where the birth functions are assumed differentiable and

$$
\kappa=-b_{m}^{\prime}\left(\tilde{S}_{m}\right)+\mu_{m}+\frac{\beta_{c m} \tilde{I}_{c}}{N_{c}},
$$


and of the matrix

$$
\left[\begin{array}{cc}
b_{s}^{\prime}(0)-\frac{\beta_{m s} \tilde{I}_{m}}{N_{c}}-\mu_{s} & \gamma \\
\frac{\beta_{m s} \tilde{I}_{m}}{N_{c}} & -\left(\gamma+\mu_{i s}\right)
\end{array}\right] .
$$

The eigenvalues of the second matrix have negative real parts if and only if

$$
0<-b_{s}^{\prime}(0)+\mu_{s}+\frac{\beta_{m s} \tilde{I}_{m}}{N_{c}} \frac{\mu_{i s}}{\left(\gamma+\mu_{i s}\right)} .
$$

Here $\frac{\mu_{i s}}{\gamma+\mu_{i s}}$ is an infected sheep's probability of dying during the infection stage [28, Sec. 2.3]. Since $\tilde{I}_{m}$ is independent of the sheep parameter, this condition can be satisfied by choosing $\beta_{m s}$ large enough.

The eigenvalues of $J$ all have negative real parts if the trace $\operatorname{tr} J$ and the determinant $\operatorname{det} J$ of $J$ are negative and

$$
\hbar J:=\operatorname{det} J-(\operatorname{tr} J)\left(\sum_{i=1}^{3} J_{i i}\right)>0
$$

where $J_{i i}$ is the determinant of the $2 \times 2$ matrix formed from $J$ by deleting the $i^{\text {th }}$ row and column; see, for example, [8, Thm.12]. By (4.13),

$$
\operatorname{tr} J=-\frac{\beta_{m c} \tilde{I}_{m}}{N_{c}}-\mu_{c}-\kappa-\mu_{i m}
$$

It is reasonable to assume that the per capita midge birth rate $\frac{b_{m}(x)}{x}$ is a decreasing function of midge density $x$, which implies that $b_{m}^{\prime}\left(\tilde{S}_{m}\right) \leq \frac{b_{m}\left(\tilde{S}_{m}\right)}{\tilde{S}_{m}}$. Then the second equation in (4.9) implies that $\kappa \geq 0$ and $\operatorname{tr} J<0$. By (4.10),

$$
\begin{aligned}
\operatorname{det} J & =-\frac{\beta_{m c} \tilde{I}_{m}}{N_{c}} \kappa \mu_{i m}-\frac{\beta_{c m} \tilde{I}_{c}}{N_{c}} \mu_{c} \mu_{i m}<0, \\
\sum J_{i i} & =\left(\frac{\beta_{m c} \tilde{I}_{m}}{N_{c}}+\mu_{c}+\mu_{i m}\right) \kappa+\frac{\beta_{m c} \tilde{I}_{m}}{N_{c}} \mu_{i m} .
\end{aligned}
$$

Since $\kappa \geq 0$, by (4.16),

$$
\hbar J \geq-\frac{\beta_{c m} \tilde{I}_{c}}{N_{c}} \mu_{c} \mu_{i m}+\frac{\beta_{m c} \tilde{I}_{m}}{N_{c}} \mu_{i m}\left(\frac{\beta_{m c} \tilde{I}_{m}}{N_{c}}+\mu_{c}+\mu_{i m}\right) .
$$

The first equation of (4.9) gives $\mu_{c} \tilde{I}_{c} \leq \beta_{m c} \tilde{I}_{m}$ and so

$$
\hbar J \geq \mu_{i m} \frac{\beta_{m c} \tilde{I}_{m}}{N_{c}}\left(-\beta_{c m}+\frac{\beta_{m c} \tilde{I}_{m}}{N_{c}}+\mu_{c}+\mu_{i m}\right) .
$$

In particular, $\hbar J>0$ if $\mu_{c}+\mu_{i m} \geq \beta_{c m}$. This condition is compatible with $\tilde{\mathcal{R}}_{0}>$ 1 by choosing $\frac{\beta_{m c} S_{m}^{0}}{N_{c}}$ large enough. This implies that the equilibrium is locally asymptotically stable [8, Thm.12].

Summarizing the above discussion gives the following result which means that, at this equilibrium, the disease can wipe out any sheep population that is introduced in small numbers.

Theorem 6. Assume that the per-capita midge birth rate $b_{s}(x) / x$ is a decreasing function of midge density $x$. If

$$
\mu_{c}+\mu_{i m} \geq \beta_{c m}, \quad 1<\frac{\beta_{m c}}{\mu_{i m}} \frac{\beta_{c m} S_{m}^{0}}{\mu_{c} N_{c}}=\tilde{\mathcal{R}}_{0} \quad \text { and } \quad b_{s}^{\prime}(0)<\mu_{s}+\frac{\beta_{m s} \tilde{I}_{m}}{N_{c}} \frac{\mu_{i s}}{\left(\gamma+\mu_{i s}\right)},
$$

then there exists a disease equilibrium with midges and cattle, but no sheep, which is locally asymptotically stable for the epidemic system involving all three species. 
5. Discussion. We have derived and analyzed a mathematical model for the time evolution of bluetongue disease in sheep and cattle. The model takes the form of a coupled system of differential equations incorporating two time delays that model the maturation time for the midges and the incubation time of the virus in the midges.

We have established fundamental properties of the model, obtained necessary and sufficient conditions for local stability of the disease free equilibrium and for disease uniform weak persistence in the midges, and sufficient conditions for global stability of the disease free equilibrium. We also studied an important particular case in which the incubation period is exponentially distributed. Since the disease affects sheep more than cattle, we incorporated recovery and disease-induced death for the sheep and assumed that only healthy sheep reproduce. As a consequence, the disease can wipe out the sheep under certain circumstances. Mathematically, this makes it difficult to prove the more desirable result of uniform disease persistence where the limit superior is replaced by the limit inferior in Theorem 4, a result that we conjecture, but that remains open at this point.

We have identified a basic reproduction number $\mathcal{R}_{0}$, given by (3.16). If the disease is introduced at a low level, it will die out if $\mathcal{R}_{0}<1$ holds, whereas it will persist if the opposite inequality $\mathcal{R}_{0}>1$ holds. These inequalities can be interpreted in a variety of ways and give guidance for control strategies. Inequality $\mathcal{R}_{0}<1$ is satisfied if at least one of the contact rates involving cattle and at least one of those involving sheep is sufficiently low, if the mortality $\mu_{i m}$ of infected midges is sufficiently high or if there is a low probability that a midge, having acquired the virus from a blood meal, will survive the incubation period to become an infectious midge (this probability is $\int_{0}^{\infty} f(\eta) \exp \left(-\mu_{e m} \eta\right) d \eta$ ). The inequality $\mathcal{R}_{0}<1$ is also satisfied if $S_{m}^{0}$ is low, and for reasonable birth functions the quantity $S_{m}^{0}$ decreases as $\mu_{l}, \mu_{m}$ or $\tau$ increases, i.e. per-capita larval, adult susceptible midge mortality or midge maturation delay increases. Moreover, $\mathcal{R}_{0}<1$ is satisfied whenever $S_{s}^{0}$ or $N_{c}$ is sufficiently high. It is slightly less obvious why this should be so, and it is a conclusion that can only be drawn from the linearized analysis and therefore for low level introductions of disease (compare with the involvement of $S_{s}^{0}$ and $N_{c}$ in the inequality for global stability, inequality (3.26)). It comes about because of the assumption of frequency-dependent transmission rather than simple mass action and is to do with the lowering of certain probabilities as $S_{s}^{0}$ or $N_{c}$ are increased. For example, increasing $N_{c}$ lowers the probability that an individual bite by an infectious midge is on a susceptible sheep (this probability being close to $S_{s}^{0} /\left(S_{s}^{0}+N_{c}\right)$ near the disease free equilibrium), although increasing $N_{c}$ increases the probability, approximately $N_{c} /\left(S_{s}^{0}+N_{c}\right)$, that the bitten animal is a cow. However, as with any vector-borne disease, transmission is a two-stage process that requires a susceptible midge to acquire the virus by biting an infectious animal, and then later to pass on the virus when it bites as an infectious midge. Increasing $N_{c}$ lowers the probability, when a susceptible midge bites, that the bitten animal is infectious, and this applies to both cows and sheep. The probabilities here are respectively roughly $I_{c} /\left(S_{s}^{0}+N_{c}\right)$ and $I_{s} /\left(S_{s}^{0}+N_{c}\right)$ at the disease free equilibrium. The effect of increasing $S_{s}^{0}$ is similar. The threshold parameter, $\mathcal{R}_{0}$, is strictly decreasing in $\mu_{c}$ (per-capita cattle mortality) and in $\gamma+\mu_{i s}$ (the sum of the per-capita recovery rate and mortality rate of infectious sheep).

For the case that the incubation period is exponentially distributed, we also calculated type reproduction numbers, which focus on one host type and provide guidance for targeted control of this one type. For example, the proportion of midges that must be eliminated to eliminate bluetongue disease is given in terms of the basic reproduction number.

Spatial effects have not been considered in this model, but the northward expansion of bluetongue across Europe suggests that the incorporation of spatial effects 
is very important and might facilitate, for example, a theoretical prediction of the spatial spread rate of bluetongue. However, the simple random walk is likely to be too oversimplistic as a model for midge dispersal due to their well known swarming tendency. This point is discussed in Okubo and Levin [24], where the use of a diffusion-advection equation is suggested, involving an advection velocity that varies within a swarm. It is also well known that environmental features such as trees, animals and water surfaces can act as swarm markers. Theoretical study of the effects of vaccination will also be an important area of future study.

Acknowledgement. We would like to thank the referees for their comments that have helped to improve the paper.

\section{REFERENCES}

[1] American Veterinary Medical Association. Bluetongue Backgrounder, 2006. Available from www.avma.org

[2] R.M. Anderson and R.M. May, Infectious diseases of humans. Oxford University Press, London/New York, 1991.

[3] F.V. Atkinson and J.R. Haddock, On determining phase spaces for functional-differential equations. Funkcial. Ekvac. 31 (1988), pp. 331-347.

[4] K.R. Bonneau, C.D. DeMaula, B.A. Mullens and N.J. MacLachlan, Duration of viraemia infectious to Culicoides sonorensis in bluetongue virus-infected cattle and sheep. Veterinary Microbiology 88 (2002), pp. 115-125.

[5] C. Bowman, A.B. Gumel, P. van den Driessche, J. Wu and H. Zhu, A mathematical model for assessing control strategies against West Nile virus. Bull. Math. Biol. 67 (2005), pp. 1107-1133.

[6] S. Busenberg, and K.L. Cooke, The effect of integral conditions in certain equations modelling epidemics and population growth, J. Math. Biol. 10 (1980), pp. 13-32

[7] Defra, UK - Animal health and welfare - Bluetongue disease. Available from www.defra.gov.uk

[8] T. Dhirasakdanon and H.R. Thieme, Stability of the endemic coexistence equilibrium for one host and two parasites, Math. Model. Nat. Phenom 5 (2010), pp. 109-138

[9] O. Diekmann and J.A.P. Heesterbeek, Mathematical epidemiology of infectious diseases: model building, analysis and interpretation. Wiley, Chichester, 2000.

[10] E.P.J. Gibbs and E.C. Greiner, The epidemiology of bluetongue, Comparative Immunology, Microbiology and Infectious Diseases. 17 (1994), pp. 207-220.

[11] S. Gubbins, S. Carpenter, M. Baylis, J.L.N. Wood and P.S. Mellor, Assessing the risk of bluetongue to UK livestock: uncertainty and sensitivity analysis of a temperature-dependent model for the basic reproduction number. J. R. Soc. Interface. 5 (2008), pp. 363-371.

[12] J.K. Hale, Functional Differential Equations, Springer, New York 1971

[13] N.A. Hartemink, B.V. Purse, R. Meiswinkel, H.E. Brown, A. de Koeijer, A.R.W. Elbers, G.-J. Boender, D.J. Rogers and J.A.P. Heesterbeek, Mapping the basic reproduction number $\left(R_{0}\right)$ for vector-borne diseases: A case study on bluetongue virus, Epidemics 1 (2009), pp. 153-161.

[14] J.A.P. Heesterbeek and M.G. Roberts, The type-reproduction number $T$ in models for infectious disease control. Math. Biosci. 206 (2007), pp. 3-10.

[15] Y. Hino, S Murakami, T Naito, Functional differential equations with infinite delay. Lecture Notes in Mathematics 1473, Springer-Verlag, Berlin/New York. 1991.

[16] W.M. Hirsch, H. Hanisch, J.-P. Gabriel, Differential equation models for some parasitic infections: methods for the study of asymptotic behavior. Comm. Pure Appl. Math. 38 (1985), pp. 733-753.

[17] J.L. Hourrigan and A.L. Klingsporn, Bluetongue: the disease in cattle. Aust. Vet. J. 51 (1975), pp. 170-174.

[18] W. Huang, K.L. Cooke and C. Castillo-Chavez, Stability and bifurcation for a multiple-group model for the dynamics of HIV/AIDS transmission. SIAM J. Appl. Math. 52 (1992), pp. 835-854.

[19] J. Jiang, Z. Qiu, J. Wu and H. Zhu, Threshold conditions for West Nile virus outbreaks, Bull. Math. Biol. 71 (2009), pp. 627-647.

[20] Y. Kuang, Delay differential equations with applications in population dynamics. Mathematics in Science and Engineering, 191. Academic Press, Inc., Boston, MA, 1993.

[21] M. Martcheva, H.R. Thieme, Progression age enhanced backward bifurcation in an epidemic model with superinfection, J. Math. Biol. 46 (2003), pp. 385-424

[22] P.S. Mellor, M. Baylis, P.P.C. Mertens (Eds). Bluetongue. Biology of Animal Infections. Academic Press 2009.

[23] Merck Veterinary Manual, http://www.merckvetmanual.com 
[24] A. Okubo and S.A. Levin, Diffusion and ecological problems: modern perspectives. Second edition. Interdisciplinary Applied Mathematics, 14. Springer-Verlag, New York, 2001.

[25] M.G. Roberts and J.A.P. Heesterbeek, A new method for estimating the effort required to control an infectious disease, Proc. Roy. Soc. Lond. B. 270 (2003), pp. 1359-1364.

[26] W.M. Ruess and W.H. Summers, Linearized stability for abstract differential equations with delay, J. Math. Anal. Appl. 198 (1996), pp. 310-336.

[27] H.L. Smith, Monotone dynamical systems. An introduction to the theory of competitive and cooperative systems. Mathematical Surveys and Monographs, 41. American Mathematical Society, Providence, RI, 1995.

[28] H.R. Thieme, Mathematics in population biology. Princeton Series in Theoretical and Computational Biology. Princeton University Press, Princeton, NJ, 2003.

[29] P. van den Driessche and J. Watmough, Reproduction numbers and sub-threshold endemic equilibria for compartmental models of disease transmission. Math. Biosci. 180 (2002), pp. 29-48.

[30] A.J. Wilson and P.S. Mellor. Bluetongue in Europe: past, present and future. Phil. Trans. R. Soc. B. 364 (2009), pp. 2669-2681.

[31] M.J. Wonham, T. de-Camino Beck and M.A. Lewis, An epidemiological model for West Nile virus: Invasion analyis and control applications. Proc. Roy. Soc. Lond. B. 271 (2004), pp. 501-507. 